\title{
THE ROLE OF BEAM GEOMETRY IN POPULATION STATISTICS AND PULSE PROFILES OF RADIO AND $\gamma$-RAY PULSARS
}

\author{
Peter L. Gonthier \\ Hope College, Department of Physics and Engineering, 27 Graves Place \\ Holland, MI 49423-9000 \\ gonthier@hope.edu \\ Robert Van Guilder \\ University Colorado at Denver, Department of Physics, Denver, CO 80217 \\ rvanguilder@usa.net \\ and \\ Alice K. Harding \\ NASA Goddard Space Flight Center, Laboratory for High Energy Astrophysics \\ Greenbelt, $M D$ 20771 \\ harding@twinkie.gsfc.nasa.gov
}

\begin{abstract}
We present results of a pulsar population synthesis study that incorporates a number of recent developments and some significant improvements over our previous study. We have included the results of the Parkes multi-beam pulsar survey in our select group of nine radio surveys, doubling our sample of radio pulsars. More realistic geometries for the radio and $\gamma$-ray beams are included in our Monte Carlo computer code that simulates the characteristics of the Galactic population of radio and $\gamma$-ray pulsars. We adopted with some modifications the radio beam geometry of Arzoumanian, Chernoff \& Cordes (2002). For the $\gamma$-ray beam, we have assumed the slot gap geometry described in the work of Muslimov $\&$ Harding (2003). To account for the shape of the distribution of radio pulsars in the $\dot{P}-P$ diagram, we continue to find that decay of the magnetic field on a timescale of $2.8 \mathrm{Myr}$ is needed. With all nine surveys, our model predicts that EGRET should have seen 7 radio-quiet (below the sensitivity of these radio
\end{abstract}


surveys) and 19 radio-loud $\gamma$-ray pulsars. AGILE (nominal sensitivity map) is expected to detect 13 radio-quiet and 37 radio-loud $\gamma$-ray pulsars, while GLAST, with greater sensitivity is expected to detect 276 radio-quiet and 344 radio-loud $\gamma$-ray pulsars. When the Parkes multi-beam pulsar survey is excluded, the ratio of radio-loud to radio-quiet $\gamma$-ray pulsars decreases, especially for GLAST. The decrease for EGRET is 45\%, implying that some fraction of EGRET unidentified sources are radio-loud $\gamma$-ray pulsars. In the radio geometry adopted, short period pulsars are core dominated. Unlike the EGRET $\gamma$-ray pulsars, our model predicts that when two $\gamma$-ray peaks appear in the pulse profile, a dominant radio core peak appears in between the $\gamma$-ray peaks. Our findings suggest that further improvements are required in describing both the radio and $\gamma$-ray geometries.

Subject headings: radiation mechanisms: non-thermal — magnetic fields - stars: neutron - pulsars: general $-\gamma$ rays: theory

\section{Introduction}

Rotation-powered pulsars are the brightest class of $\gamma$-ray sources detected by the Compton $\gamma$-Ray Observatory (CGRO). The high-energy telescope EGRET made firm detections of pulsed $\gamma$-ray emission from five known radio pulsars (Thompson 2001), and possible detections from several others (Kanbach 2002). In addition, the high-energy pulsar Geminga may be radio-quiet, or at least radio-weak. EGRET also discovered more than $200 \gamma$-ray sources (Hartman et al. 1999), most of which are still unidentified. However, several dozen new radio pulsars, out of more than 600 discovered since the end of the CGRO mission by the recent Parkes multi-beam pulsar survey (PMBPS) (Manchester et al. 2001) or in deep targeted observations (Lorimer 2003), lie within the error circles of EGRET sources. Although many of these are young, energetic pulsars, their identification as $\gamma$-ray pulsars must await observation with the next $\gamma$-ray telescopes, AGILE and GLAST.

In the meantime, population synthesis studies of radio and $\gamma$-ray pulsars can predict the number of radio-loud and radio-quiet $\gamma$-ray pulsar detections expected by different telescopes, assuming different models for radio and $\gamma$-ray emission. Even though current radio and $\gamma$-ray emission models have a number of outstanding uncertainties, the results of such studies can provide quite sensitive discrimination between models. In particular, polar cap and outer gap models of $\gamma$-ray emission make very different predictions of the number of radio-loud and radio-quiet $\gamma$-ray pulsars. Polar cap models (e.g. Daugherty \& Harding 1996), where the high-energy emission region is located on the same open field lines as the radio emission, expect a large overlap in the radio and $\gamma$-ray emission beams and thus a higher ratio of radio- 
loud to radio-quiet $\gamma$-ray pulsars. On the other hand, outer gap models predict a smaller overlap between $\gamma$-ray and radio emission beams, because the high-energy and visible radio emission originate from opposite poles (Romani \& Yadigaroglu 1995, Cheng et al. 2000), thus predicting more radio-quiet than radio-loud $\gamma$-ray pulsars. Thus, population synthesis can also address the question of how many EGRET unidentified sources are radio pulsars.

Results of our initial study of pulsars in the Galaxy were presented by Gonthier et al. (2002). In this work, we evolved neutron stars from birth distributions in space, magnetic field strength, period and kick velocity, in the Galactic potential to simulate the population of radio pulsars detected in eight surveys of the Princeton catalog (Taylor, Manchester \& Lyne 1993). A very simple model of radio and $\gamma$-ray beams was assumed, in which both were aligned with solid angle of $1 \mathrm{sr}$. Radio luminosity was assigned using the model of Narayan \& Ostriker (1990) and $\gamma$-ray luminosity from the polar cap model of Zhang \& Harding (2000). We found that agreement of the distribution of simulated radio pulsars with the observed distribution was significantly improved by assuming decay of the neutron star surface magnetic field on a time scale of $5 \mathrm{Myr}$. With these assumptions, EGRET should have detected 9 radio-loud and 2 radio-quiet $\gamma$-ray pulsars, and GLAST should detect 90 radio-loud and 101 radio-quiet pulsars (9 detected as pulsed sources). Because the radio and $\gamma$-ray beam apertures were assumed to be identical, radio-quiet $\gamma$-ray pulsars were those whose radio emission is too weak to be detected by the selected radio surveys.

There have been a number of new developments in both radio pulsar observation and analysis, and in $\gamma$-ray pulsar theory since we completed our initial population study. The PMBPS (Manchester et al. 2001, Morris et al. 2002, Kramer et al. 2003) is nearly complete and has more than doubled the number of radio pulsars with measured period derivatives from 445 to nearly 1300. Determination of pulsar distances from dispersion measure has been greatly improved with the development of a new model by Cordes \& Lazio (2002). New radio luminosity and beam models have been developed by Arzoumanian, Chernoff \& Cordes (2002), which describe core and conal components of the emission and their dependence on period and frequency. Arzoumanian, Chernoff \& Cordes (2002) have also derived a new two-component distribution of radio pulsar space velocities. A new polar cap $\gamma$-ray emission model has been developed by Muslimov \& Harding (2003), in which radiation from pair cascades at high altitude along the edge of a slot gap forms a wide hollow cone of emission. In addition, the solid angle as well as the luminosity of the $\gamma$-ray beam is described in this model.

This paper presents results of an expanded and updated pulsar population synthesis study that includes all of the above recent developments, as well as improved $\gamma$-ray sensitivity maps. By incorporating independent models for the radio and $\gamma$-ray beam geometry, we are 
now able to investigate how the beam geometry affects the observable characteristics of radioloud and radio-quiet $\gamma$-ray pulsar populations. We are also able to address the question of how many EGRET unidentified sources are expected to be radio-loud or radio-quiet $\gamma$-ray pulsars in the polar cap model. Of particular interest is the issue of how many of the new Parkes radio pulsars in EGRET error circles are counterpart $\gamma$-ray pulsars. In addition, we can make more accurate estimates of the numbers of radio-loud or radio-quiet $\gamma$-ray pulsars detectable by the AGILE and GLAST telescopes.

\section{Radio Emission Geometry}

We have adopted the geometry model for the radio emission beams as presented by Arzoumanian, Chernoff \& Cordes (2002) (ACC from now on) with some slight modifications. We have assumed a core and a single conal component described by Gaussians with characteristic widths as follows

$$
\begin{aligned}
& \rho_{\text {core }}=1.5^{\circ} P^{-0.5}, \text { and } \\
& \rho_{\text {cone }}=5.2^{\circ}\left(1+\frac{66}{\nu}\right) P^{-0.5}
\end{aligned}
$$

where the period, $P$, is in seconds and the frequency, $\nu$, is in $\mathrm{MHz}$. The characteristic core width is the width of the core beam at $1 / e$ of the peak intensity. We have incorporated the radius-to-frequency mapping in the conal width developed by Mitra \& Deshpande (1999); although they introduce elliptical shapes to the conal geometry, they find no compelling reason to abandon circular beams. The coefficient of $5.2^{\circ}$ above is chosen to give the same width at $400 \mathrm{MHz}$ as in ACC.

For each simulated pulsar, the pulse profile is binned into 500 bins of the phase angle, $\phi$, ranging from $-\pi$ to $\pi$. Each bin is assigned a flux, $s(\phi, \nu)$, consisting of the sum of the flux contributions from the core and cone components given by

$$
\begin{aligned}
s(\phi, \nu) & =S_{\text {core }}(\theta, \nu)+S_{\text {cone }}(\theta, \nu) \\
S_{i}(\theta, \nu) & =-\frac{\alpha_{i}+1}{\nu}\left(\frac{\nu}{50 \mathrm{MHz}}\right)^{\alpha_{i}+1} f_{i}(\theta) \frac{L_{i}}{d^{2}}
\end{aligned}
$$

where $i$ indicates core or cone, $\alpha_{i}$ is the spectral index $\left(S_{i} \propto \nu^{\alpha_{i}}\right.$ with $\left.\alpha_{i}<0\right)$, $\theta$ is the polar angle to the magnetic axis, $f_{i}(\theta)$ is the angular distribution of the component flux, $L_{i}$ 
is the component luminosity and $d$ is the distance to the pulsar. The relationship between the phase angle, $\phi$, and the polar angle, $\theta$, depends on the viewing geometry given by the expression

$$
\cos \theta=\sin \alpha \sin \zeta \cos \phi+\cos \alpha \cos \zeta
$$

During the simulation, the magnetic inclination angle, $\alpha$, and the observer's line of sight angle, $\zeta$, are chosen randomly between zero and $\pi / 2$, accounting for emission from both poles. The difference between these two angles defines the impact angle, $\beta=\zeta-\alpha$.

We assume that the spectrum of each component has a low frequency cutoff of 50 $\mathrm{MHz}$ and can be modeled by a single power law, with spectral indices of $\alpha_{\text {core }}=-2.1$ and $\alpha_{\text {cone }}=-1.6$. It is general agreed that the spectra of cores are steeper that those of cones especially for short-period pulsars (Lyne \& Manchester 1988 and Rankin 1983). As discussed later in section 5 , we have assumed constant spectral indices with a difference of 0.5 between the core and cone indices.

The angular distributions of the core and conal components are given by the Gaussians

$$
\begin{aligned}
& f_{\text {core }}(\theta)=\frac{1}{\Omega_{\text {core }}} e^{-\theta^{2} / \rho_{\text {core }}^{2}}, \text { and } \\
& f_{\text {cone }}(\theta)=\frac{1}{\Omega_{\text {cone }}} e^{-(\theta-\bar{\theta})^{2} / w_{e}^{2}} .
\end{aligned}
$$

The solid angles for each of the components are chosen to normalize the Gaussian distributions describing the angular distribution of the flux in equation (5) when integrating over the polar angle, $\theta$, the azimuthal angle, $\phi$, and are given by the approximate expressions

$$
\begin{aligned}
& \Omega_{\text {core }}=\pi \rho_{\text {core }}^{2}, \text { and } \\
& \Omega_{\text {cone }}=2 \pi^{3 / 2} w_{e} \bar{\theta} \sim 0.8 \pi \rho_{\text {cone }}^{2}
\end{aligned}
$$

The width and radius of the annulus of the conal beam are given by

$$
\begin{aligned}
w_{e} & =\frac{\rho_{\text {cone }}}{4 \sqrt{\ln 2}}, \text { and } \\
\bar{\theta} & =0.75 \rho_{\text {cone }}=3.9^{\circ}\left(1+\frac{66}{\nu}\right) P^{-0.5}
\end{aligned}
$$


These expression differ slightly from the ones used by Arzoumanian (private communication) given by the following forms:

$$
\begin{aligned}
& \Omega_{\text {core }}^{A C C}=\frac{\pi \rho_{\text {core }}^{2}}{\ln 2} \sim 1.4 \pi \rho_{\text {core }}^{2}, \text { and } \\
& \Omega_{\text {cone }}^{A C C}=\frac{2 \pi \rho_{\text {cone }}^{2}}{5 \ln 2} \sim 0.58 \pi \rho_{\text {cone }}^{2} .
\end{aligned}
$$

With the above definition of $w_{e}$ when $\theta=\rho_{\text {cone }}, \rho_{\text {cone }}$ represents the radius of the cone at half power. The half-angle coefficient of $3.9^{\circ}$ in equation (6) is the angle where the conal intensity peaks. This coefficient corresponds to that of the middle cone of the three conal components discussed by Mitra \& Deshpande (1999). The phase angle, $\phi$, is varied between $-\pi$ and $\pi$ and divided into 500 bins with the flux contributions of core and conal components evaluated and summed. In order to see if the simulated pulsar is detected, the averaged flux, $S_{a v e}$, of the pulse profile is then compared to the flux threshold, $S_{\text {min }}$, of each survey at its corresponding frequency. If detected, the pulsar is flagged as radio-loud otherwise it is radio-quiet.

In the ACC study, pulsar surveys were all selected near a frequency of $400 \mathrm{MHz}$; hence they had no need to introduce any frequency dependence to the fluxes of the core and conal components. An important assumption made in ACC is that the ratio of the core to cone flux is given by $r=\frac{20}{3} P^{-1}$. In the present study, we have two groups of pulsar surveys. One group has frequencies near $400 \mathrm{MHz}$, while the other group has frequencies near $1400 \mathrm{MHz}$. Therefore, we have had to introduce the above frequency dependence to the spectra of the core and conal components with a ratio of the core to cone peak flux given by

$$
r=10 P^{-1}\left(\frac{\alpha_{\text {core }}+1}{\alpha_{\text {cone }}+1}\right)\left(\frac{\nu}{400 \mathrm{MHz}}\right)^{\alpha_{c o r e}-\alpha_{\text {cone }}},
$$

where this ratio is similar to the ratio used in $\mathrm{ACC}$ at a constant frequency of $400 \mathrm{MHz}$. With this ratio, short period pulsars will have their radio fluxes dominated by the core component with a weak conal component depending on the viewing geometry.

The luminosities of the core and cone components are expressed by

$$
\begin{aligned}
& L_{\text {cone }}=\frac{L}{\left(1+r / r_{o}\right)}, \text { and } \\
& L_{\text {core }}=\frac{L}{\left(1+r_{o} / r\right)}, \text { where }
\end{aligned}
$$




$$
r_{o}=\frac{\Omega_{\text {cone }}}{\Omega_{\text {core }}}\left(\frac{\alpha_{\text {core }}+1}{\alpha_{\text {cone }}+1}\right)\left(\frac{\nu}{50 \mathrm{MHz}}\right)^{\alpha_{\text {core }}-\alpha_{\text {cone }}}
$$

where $r / r_{o}$ is the ratio of core to cone luminosities, and $L$ is the total luminosity given by

$$
L=3.4 \times 10^{10} P^{-1.3} \dot{P}^{0.4}\left(\mathrm{mJy} \cdot \mathrm{kpc}^{2} \cdot \mathrm{MHz}\right) .
$$

This luminosity is reduced by a factor of 60 from the one used in ACC, as discussed in section 5. Under this assumption, radio pulsars are believed to be standard candles with well-defined luminosities in terms of only the period and period derivative whose exponents in the expression above for $L$ come from parameters in Table 1 of ACC for the first model assuming a braking index $n=3$ with two velocity components. There is no dithering of the luminosity as in the case of Narayan \& Ostriker (1990). Here the random viewing geometry accounts completely for the required dithering when the beam and viewing geometries are not included. However as discussed later in the text in section 5 , in order to obtain a resonable birth rate and adequate agreement between the distributions of the distance, flux and dispersion measure, we have had to reduce the radio luminosity used in ACC by a substantial amount.

\section{Gamma-ray Emission Geometry}

For the geometry of the $\gamma$-ray beam, we have adapted the emission from the slot gap described in Muslimov \& Harding (2003). The slot gap (Arons \& Scharlemann 1979) is a narrow region between two conducting boundaries, the last open field line and the pair formation front, extending from the neutron star surface up to the light cylinder. Since the electric field is relatively small in the slot gap, primary particles accelerate more slowly and pair cascades form at altitudes of several stellar radii above the surface. We model the radiation from these pair cascades as having two components, curvature radiation of the primary electrons and synchrotron radiation from the electron-positron pairs. We obtain the number of curvature, $N_{C R}$, and synchrotron, $N_{S R}$, photons emitted per primary particle by integrating the differential production rates over the available energy and over the pulsar phase angle $\phi$. Since we have not included relativistic effects such as aberration and timeof-flight delays in modeling the $\gamma$-ray beam, the caustic peaks as found by Dyks \& Rudak (2003) and in outer gap models (e.g. Romani \& Yadigaroglu 1995) do not appear in our calculations. 


\subsection{Curvature Radiation}

We assume that the curvature radiation takes place in the slot gap at the last open field line (see Figure 1) and is integrated from a lower $\gamma$-ray threshold, $E_{\gamma}$, of $100 \mathrm{MeV}$ to the curvature radiation critical energy, where $E_{\gamma}$ and $E_{C R}$, are in $m_{e} c^{2}$ units and having an angular distribution represented by the expression

$$
\begin{aligned}
\frac{d N_{C R}\left(>E_{\gamma}, \theta_{\gamma}\right)}{d \Omega} & =\frac{3 e^{2}}{2 \pi \hbar c \sin \theta}\left(\frac{\rho m_{e} c}{\hbar}\right)^{1 / 3}\left[E_{C R}^{1 / 3}-E_{\gamma}^{1 / 3}\right], \text { where } \\
E_{C R} & =\frac{3 \gamma_{o}^{3} \hbar}{m_{e} c \rho}\left[1+\frac{9 \pi r_{e} \gamma_{o}^{3}}{4 c P} \ln \left(\frac{r}{R}\right)\right]^{-1}, \text { is the curvature photon critical energy and } \\
\rho & =\frac{r\left(1+\cos ^{2} \theta\right)^{3 / 2}}{3 \sin \theta(1+\cos \theta)}, \text { is the radius of curvature with } \\
r & =\frac{c P}{2 \pi} \sin ^{2} \theta
\end{aligned}
$$

where $r$ is the radial distance on the last open dipole field line corresponding to magnetic colatitude, $\theta$, and $\theta_{\gamma}=\frac{3}{2} \theta$ is the photon emission angle, which is tangent to this field line. $R$ is the neutron star radius taken to be $10^{6} \mathrm{~cm}, r_{e}$ is the Compton wavelength of an electron and $\gamma_{o}$ is the initial Lorentz factor of the particle given by

$$
\begin{aligned}
& \gamma_{o}=\frac{10^{6} B_{12}}{P^{2}}, \text { for } B_{12}^{16 / 7} P^{-27 / 7}<1440, \text { or } \\
& \gamma_{o}=4 \times 10^{7} P^{1 / 14} B_{12}^{-1 / 7}, \text { for } B_{12}^{1 / 7} P^{-27 / 7}>3.0 \text { in regime I } \\
& \gamma_{o}=1.4 \times 10^{7} P^{-1 / 4}, \text { for } B_{12}^{1 / 7} P^{-27 / 7}<3.0 \text { in regime II. }
\end{aligned}
$$

where the first expression is for the case where the electric field is not screened by electronpositron pairs (Harding et al. 2002) and the second and third expressions are for cases where the electric field is screened by pairs in the unsaturated (I) and saturated (II) regimes (Zhang \& Harding 2000). $P$ is the period in seconds and $B_{12}$ is the magnetic field at the surface in units of $10^{12} \mathrm{G}$. At a given inclination angle $\alpha$, the line of sight angle, $\zeta$, and phase angle, $\phi$, define a polar angle, $\theta$ (through equation [3]), where the emission occurs tangent to the last open field line at a radial distance, $r$, from the stellar center, and the curvature emission rate per primary particle is given by equation (11) for $d N_{C R} / d \Omega$. 


\subsection{Synchrotron Radiation}

The synchrotron radiation from cascade pairs takes place along the slot gap, beginning at an altitude $R_{m i n}$, where the first pairs are produced, and continuing out to $R_{S R}$, the maximum radius at which pairs are produced (see Figure 1). $R_{S R}$ is determined as the altitude where the curvature radiation critical energy, $E_{C R}$, is equal to the photon escape energy, $E_{\text {esc }}$ (i.e. the minimum energy of photons capable of one-photon pair production). The pair-escape energy, $E_{e s c}(r)$, in $m_{e} c^{2}$ units, is given approximately by (see Zhang \& Harding 2000 or Harding 2001)

$$
E_{e s c}(r) \approx 518\left(\frac{r P}{R}\right)^{1 / 2} \max \left(1, \frac{0.1}{B^{\prime}}\right)
$$

The angles $\theta_{\min }$ and $\theta_{s}$ are those corresponding to the radii $R_{m i n}$ and $R_{S R}$ along the field line at the edge of the slot gap,

$$
\begin{aligned}
\theta_{\min } & =\sin ^{-1}\left(\frac{2 \pi R_{\min }}{c P}\right), \\
\theta_{s} & =\sin ^{-1}\left(\frac{2 \pi R_{S R}}{c P}\right) .
\end{aligned}
$$

The corresponding photon emission angles are $\theta_{\gamma, \min } \approx \frac{3}{2} \theta_{\min }$ and $\theta_{\gamma, s} \approx \frac{3}{2} \theta_{s}$. The parameter, $R_{\min } / R$ is set to 3.5 in all of our simulations, fixing the beginning of the emission zone to be 2.5 stellar radii above the surface (Muslimov \& Harding 2003). We assume that the electron-positron pairs in the cascade have a spectrum $N_{ \pm}\left(\gamma_{p}\right)=C_{p} \gamma_{p}^{-p}$, extending from a minimum $\gamma_{\min }=E_{e s c}\left(R_{\min }\right) / 2$ to a maximum at $\gamma_{\max }=E_{C R} / 2$, where $E_{\text {esc }}\left(R_{\min }\right)$ is the photon pair-escape energy at radius $R_{\text {min }}$.

The integral photon spectrum above energy $E_{\gamma}$ of the synchrotron radiation from the electronpositron pairs with spectral index $p$, per primary particle, is

$$
\begin{aligned}
N_{S R}\left(>E_{\gamma}\right) & =\frac{2 C_{S}}{(1-p)}\left[E_{\gamma}^{(1-p) / 2}-E_{S R}^{(1-p) / 2}\right], \text { where } \\
E_{S R} & =\frac{3 \gamma_{\max }^{2} B^{\prime} \sin \psi}{2} .
\end{aligned}
$$


$E_{S R}$ is the critical synchrotron energy, in $m_{e} c^{2}$ units, of pairs at their maximum energy $\gamma_{\max }$ and $B^{\prime}$ is the local field strength in units of the critical field $4.4 \times 10^{13} \mathrm{G}$. The pitch angle $\psi$ of the pairs is assumed to be that of the parent photon direction with respect to the local field at the pair production point, or

$$
\begin{aligned}
& \sin \psi=\frac{0.1}{B^{\prime} E_{C R}}, \text { for } B^{\prime}<0.1, \text { or } \\
& \sin \psi=\frac{2}{E_{C R}}, \text { for } B^{\prime}>0.1 .
\end{aligned}
$$

The spectral index of the pairs is given by $p=2 \alpha_{n}-1$, where the spectral index of the photons, $\alpha_{n}$, is determined (Harding \& Daugherty 1999, Zhang \& Harding 2000) by the number of generations, $n$, of the pair cascade,

$$
\begin{aligned}
\alpha_{n} & =2-\frac{2-\alpha_{1}}{2^{n-1}} \\
n & =1+\frac{\ln \left(E_{e s c} / E_{o}\right)}{\ln (\kappa)}
\end{aligned}
$$

where $\kappa=3 / 64$ and $\alpha_{1}=5 / 3$ for curvature radiation with losses. The cascade generation number is determined by $E_{o}$, which is the curvature radiation critical energy at the initial colatitude angle $\theta_{\min }$ of the radiation zone.

We normalize the pair spectrum to the total cascade multiplicity

$$
N_{ \pm} \sim \frac{E_{0}}{20 E_{e s c}\left(R_{\min }\right)}
$$

so that the normalization factor $C_{S}$ for the synchrotron photon spectrum in equation (16) is given by

$$
C_{S}=\frac{4 \pi}{3.3 \sqrt{3}} \frac{2^{1-p}(1-p)}{(3 p-1)} N_{ \pm}\left[E_{0}^{(1-p)}-E_{e s c}\left(R_{\min }\right)^{(1-p)}\right] .
$$

The emission from the high altitude ( 2-4 stellar radii) cascades from the slot gap along the last open field line forms a broad, hollow-cone beam. The parameter representing the 
longitudinal thickness of the slot gap is expressed, in units of the polar cap half-angle $\theta_{\min }$, as (Muslimov \& Harding 2003)

$$
\begin{aligned}
& \Delta \xi=4 P B_{12}^{-4 / 7} \text { for } P B_{12}^{-4 / 7}<0.075 \text { or } \\
& \Delta \xi=0.3 \text { for } P B_{12}^{-4 / 7}>0.075
\end{aligned}
$$

where $B_{12}$ is in units of $10^{12} \mathrm{G}$. The acceleration-cascade simulations indicate that the width of the slot widens as the pulsar ages and saturates at a value of approximately 0.3. As seen in Figure 1, the interior and exterior polar angles of the radiation from the slot gap at $R_{\min }$ are described by the following expression

$$
\begin{aligned}
& \theta_{\min }^{S G} \sim \theta_{\gamma, \min }(1-\Delta \xi), \text { and } \\
& \theta_{\max }^{S G} \sim \theta_{\gamma, \min }
\end{aligned}
$$

We take the average opening angle of the cascade radiation from the slot gap between $r=R_{\min }$ and $r=R_{S R}$ as $\theta_{S G}=\left(\theta_{\min }^{S G}+\theta_{\gamma, s}\right) / 2$. We approximate the angular distribution of the synchrotron radiation component of the entire cascade between $\theta_{\min }$ and $\theta_{s}$ as a hollow beam with a conal Gaussian of width equal to

$$
w_{\gamma}=\theta_{\max }^{S G}-\theta_{\min }^{S G}
$$

which is the full width at $1 / e$ of the maximum. The integral photon spectrum above energy $E_{\gamma}$ of the synchrotron radiation, per primary particle at a given polar angle is then given by

$$
\frac{d N_{S R}\left(>E_{\gamma}, \theta_{\gamma}\right)}{d \Omega}=\frac{N_{S R}\left(>E_{\gamma}\right)}{\theta_{S G} \omega_{\gamma} \pi^{3 / 2}} \exp \left[-\frac{\left(\theta_{\gamma}-\theta_{S G}\right)^{2}}{\omega_{\gamma}^{2}}\right]
$$

The current of primary electrons in the slot gap that results in curvature and synchrotron radiation is limited to a fraction of the Goldreich-Julian current, $\dot{n}_{G J}$, in the following manner

$$
\dot{n}_{\text {slot gap }}=\left(1-(1-\Delta \xi)^{2}\right) \dot{n}_{G J}, \text { where }
$$




$$
\dot{n}_{G J}=\frac{5.7 \times 10^{31} B_{12}}{P^{2}}
$$

This current multiplies the integral of he curvature and synchrotron emission per primary particle to give the total slot gap emission beam.

The total flux due to both curvature and synchrotron radiation are calculated for a given phase angle, $\phi$, which is related to $\theta_{\gamma}$ through equation (3), for a pulse profile with 500 bins of phase angle from $-\pi$ to $\pi$. The average of the profile is obtained and compared to the appropriate instrumental flux threshold. If the average flux is above the threshold, the $\gamma$-ray pulsar is detected. This condition is tested independently of the radio flux and appropriate radio survey threshold, allowing us to designate the detected $\gamma$-ray pulsar as radio-loud or radio-quiet.

\section{Monte Carlo Simulations}

We discuss here some of the important changes that have been made to our Monte Carlo simulation code from our previous work in Gonthier et al. (2002). While we believe that is important to place the neutron stars at birth in spiral arms, we have not yet included the spiral arm structure into our simulations. As in Gonthier et al. (2002), we distributed pulsars at birth in the Galactic disk according to the prescription of Paczyński (1990). In a cylindrical coordinate system, the azimuthal angle, $\phi$, is randomly chosen between 0 and $2 \pi$. The $z$ distribution varies exponentially with distance from the plane, while the radial distribution peaks at $4.5 \mathrm{kpc}$ and decreases exponentially from the center of the Galaxy. Given the initial position and velocity, the trajectory of each neutron star is evolved in the Galactic potential to the present.

\subsection{Comparison group of pulsars in the ATNF catalog}

In order to have a comparison group to normalize our simulation, we have selected pulsars from the Australian Telescope National Facility (ATNF) ${ }^{1}$. We chose pulsars within the Galaxy with periods greater than $30 \mathrm{~ms}$ and with positive period derivatives to obtain a comparison group of 978 pulsars detected by these nine surveys. Selecting pulsars with

\footnotetext{
${ }^{1}$ ATNF catalog is available at: http://www.atnf.csiro.au/research/pulsar/psrcat/.
} 
periods greater than $30 \mathrm{~ms}$ insures that we have left out of our group most of the millisecond pulsars that have been recycled in binary systems. We are not currently simulating this class of pulsars since their evolution is more complicated. We have also not included the anomalous $\mathrm{X}$-ray pulsars, the soft-gamma-ray repeaters, or pulsars in globular clusters in our comparison group. We run the Monte Carlo simulation until the code detects the same total number of radio pulsars as have been observed with the group of surveys. With this normalization a neutron star birth rate is predicted as well as the number of $\gamma$-ray pulsars detected by various instruments. However, in order to obtain smoother simulated distributions, we run the code for ten times the number of pulsars detected by the radio surveys and then normalize accordingly.

\subsection{Flux sensitivity of the Parkes multi-beam pulsar survey}

We have included the eight radio surveys described in Gonthier et al. 2002 along with the new PMBPS having an angular coverage of $|b|<5^{\circ}$ and $\ell=260^{\circ}$ to $\ell=50^{\circ}$ with an assumed geometric efficiency of $100 \%$. In Gonthier et al. 2002, we calculated the minimum radio thresholds, $S_{m i n}$, for the selected group of radio surveys using the Dewey et al. (1985) formula. We attempted to use the same formula for the PMBPS using the parameters indicated in Manchester et al. (2001). However, we found that an additional factor of $\sim 2$ multiplying the limiting sensitivity is required to reproduce the $S_{\min }$ curves in Figure 2 of Manchester et al. (2001) as shown here in Figure 2. The Dewey formula under predicts the $S_{m i n}$, and a more realistic treatment of narrow pulse widths (smaller duty cycles) in the Fourier search is not as optimistic as the Dewey formula (F. Crawford private communication). As a result, we chose to evaluate the $S_{\text {min }}$ for the PMBPS using an IDL code (Crawford private communication) that we translated into $\mathrm{C}++$ to incorporate into our Monte Carlo code that is called event-by-event. This routine was used to create Figure 2 in Manchester et al. (2001) and reproduced here in Figure 2, along with the $S_{\min }$ curves predicted by the Dewey formula, with the extra factor of 2 , for the indicated DMs. In our simulations, we have then scaled the limiting sensitivities as discussed in Manchester et al. (2001).

\subsection{Gamma-ray Thresholds}

We simulate the $\gamma$-ray pulsars detected by EGRET, AGILE and GLAST. If the simulated $\gamma$-ray flux, obtained from the average flux in the pulse profile, is above a detector threshold, the pulsar is said to be a $\gamma$-ray pulsar detected by the corresponding instrument. We have included an all-sky sensitivity for both EGRET (I. Grenier, private communica- 
tion) and AGILE (A. Pellizzoni, private communication) and are shown in Figure 3a and 3b. For AGILE, we have three all-sky sensitivity maps representing the best, nominal and worst case scenarios. The one portrayed in Figure $3 \mathrm{~b}$ is for the nominal case. For GLAST, we have used the following thresholds: in-plane $\left(|b|<10^{\circ}\right) 5 \times 10^{-9}$ photons $/\left(\mathrm{cm}^{2} \cdot \mathrm{s}\right)$, outof-plane $\left(|b| \geq 10^{\circ}\right) 2 \times 10^{-9}$ photons $/\left(\mathrm{cm}^{2} \cdot \mathrm{s}\right)$ (D. Thompson private communication), and for pulsed emission $5 \times 10^{-8}$ photons $/\left(\mathrm{cm}^{2} \cdot \mathrm{s}\right.$ ) (S. Ritz private communication, McLaughlin $\&$ Cordes 2000). The above threshold for pulsed emission detection in a blind periodicity search is based on techniques used in periodicity searches of EGRET data (Mattox et al. 1996, Chandler et al. 2001).

\subsection{New distance model}

We have incorporated the new electron density model of the Galaxy from Cordes \& Lazio (2003) by calling the FORTRAN subroutines from within our code to calculate the dispersion measure (DM) of the simulated pulsar. The DM leads to a smearing of the pulse, affecting the flux threshold for radio detection. For comparison, we have recalculated the distance of the pulsars in the ATNF catalogue from the measured DM and the pulsars location using the new distance model. In Figure 4, we show the histogram for the logarithm of the absolute value of the difference between the distance obtained from the new distance model and the old distance model for our selected group of 978 pulsars. The distances obtained with the new distance model are about $20 \%$ smaller than those obtained with the old distance model. For pulsars in the catalogue whose best estimate distance is different than the one obtained using the old distance model, we have assumed that the distance was established by other methods and, therefore, is more reliable.

\subsection{Initial period distributions}

Recently various observations of young supernova remnants have been able to measure the speed of the expansion shell and the period and period derivative of the pulsar, thereby, determining the initial period of the pulsars. For example, X-ray Pulsars PSR J1811-1925 and PSR J0205+6559 (Gavriil et al. 2003) have been associated with the supernova remnants G11.2-0.3 and 3C 58, respectively, and may suggest that these pulsars where born with a period of $\sim 65 \mathrm{~ms}$. In contrast to our previous study of Gonthier et al. (2002) that used a constant birth period of $30 \mathrm{~ms}$, we studied Gaussian and flat initial spin distributions to describe the initial period. We found that the overall population statistics are not very sensitive to the initial spin distribution and only affects the short-period population of pulsars 
in the $\dot{P}-P$ diagram. While significant progress is being made in deducing the initial period of pulsars, the shape of the distribution is not well defined at the present. We have concluded that a flat distribution from 0 to $150 \mathrm{~ms}$ accommodates the observations and have used this distribution in this study.

\subsection{Decay of the Magnetic Field}

We continue to be steered in the direction of incorporating the decay of the magnetic field in order to achieve better comparisons. Originally, we included eight radio surveys in Gonthier et al. (2002) with 445 detected radio pulsars to compare to our simulated results. We used one Gaussian to describe the primary magnetic field distribution with a single decay constant. The PMBPS (Manchester et al. 2001) has discovered many more pulsars, many of which are young, distant pulsars with high radio luminosities. The current pulsar catalog now has 1412 radio pulsars (http://www.atnf.csiro.au/people/pulsar/catalogue). With the PMBPS, we have a selected group of 978 detected radio pulsars with many more high field pulsars. A single Gaussian would result in too many low field pulsars. In order to simulate these high field pulsars, we found it necessary to use two Gaussian distributions to skew the distribution towards high fields.

The pulsars surface magnetic field distribution at birth is represented by the sum of two log-normal Gaussian distributions expressed as

$$
\rho_{B}=\sum_{i=1}^{2} A_{i} e^{-\left(\log B-\log B_{i}\right)^{2} / \sigma_{i}^{2}}
$$

where the parameters are indicated in Table 1.

Table 1

\begin{tabular}{cccc}
\hline \multicolumn{4}{c}{ Parameters for Initial } \\
Magnetic Field & Distribution \\
\hline$i$ & $A_{i}$ & $\log B_{i}$ & $\sigma_{i}$ \\
\hline 1 & 0.6 & 12.75 & 0.4 \\
2 & 0.3 & 13.0 & 0.7 \\
\hline
\end{tabular}

While there are two Gaussians describing the initial field distribution of the pulsars at birth, the second Gaussian with a higher mean field merely skews the distribution towards higher magnetic fields and does not necessarily suggest two groups of pulsars with different field characteristics. Using a single broader Gaussian would result in too many lower field pulsars. 
The birth rate is assumed to be constant during the history of the Galaxy (at least back to $10^{9}$ years in the past); therefore, we randomly select the age of the pulsar from the present to $10^{9}$ years in the past. We assume a dipole spin down with a decaying magnetic field having a time constant, $\tau_{D}$, following Gonthier et al. (2002). In Figure 5, we present the period derivative versus the period of simulated pulsars with the indicated time constants from $10^{8}$ to $5 \times 10^{5}$ years. Indicated in the figure are lines of constant field (calculated according to Shapiro \& Teukolsky, 1983) and pulsar age assuming dipole spin-down of a constant field as well as the curvature radiation (CR) and nonresonant inverse Compton scattering (NRICS) death lines (Harding et al. 2002). Without field decay or a large decay constant like $10^{8}$ years, pulsars will move from their short periods to longer periods along constant or nearly constant field lines and pile up near the NRICS line.

In order to populate, without field decay, the diagram in a region of small period derivatives $\left(5 \times 10^{-18}\right)$ and medium periods $(0.5 \mathrm{~s})$, many more short period pulsars would populate the lower left region of the diagram where none are observed (in Figure 8). Decreasing the decay constant, produces the upside-down pear shaped distribution seen in the distribution of detected pulsars and populates the high field region above $5 \times 10^{13} \mathrm{G}$. Unless one can alter the period and period derivative dependence of the radio luminosity significantly in a manner with more than just a simple power law, we find that field decay is required to reproduce the distribution. In subsequent simulations, we have adopted a value of $2.8 \mathrm{Myr}$ for the decay constant.

\subsection{Supernova kick velocity distribution}

A number of studies disagree on the initial 3-D velocity distribution of neutron stars at their birth, possibly the result of an asymmetric supernova kick, typically described by a Maxwellian distribution. Lorimer, Bailes \& Harrison (1997) obtained a velocity distribution with a mean velocity of $\sim 480 \mathrm{~km} / \mathrm{s}$ similar to a previous study obtaining a mean of $\sim 450 \mathrm{~km} / \mathrm{s}$ (Lyne \& Lorimer 1994), yet significantly larger than most previous studies of pulsar statistics that required space velocities of $\sim 150 \mathrm{~km} / \mathrm{s}$. Hansen \& Phinney (1997) concluded that a mean velocity $\sim 250-300 \mathrm{~km} / \mathrm{s}$ best described their study. Though Hartman et al. (1997) did not use a Maxwellian distribution, they obtained a distribution with a mean velocity of $380 \mathrm{~km} / \mathrm{s}$. Gonthier et al. (2002) also did not use a Maxwellian distribution and found a distribution with a mean velocity of $170 \mathrm{~km} / \mathrm{s}$. It is clear that the velocity distribution that one obtains depends heavily on the many other assumptions that go into the model, such as the radio luminosity and radio beam geometry. The brighter the radio pulsars, the greater the distance at which they are detected, resulting in a broader distribution of distance, $z$, 
from the plane of the Galaxy, requiring a smaller mean velocity to improve the agreement with $z$ distribution of detected pulsars.

In this study we have adopted the luminosity model of ACC, and so we must also adopt their kick velocity model. We chose to follow their two-component velocity distribution, which is Maxwellian in velocity with characteristic widths of 90 and $500 \mathrm{~km} / \mathrm{s}$ and given by the equation (1) in ACC. In the ACC model, the two-component velocity model was preferred over the single component model. The fraction of the neutron stars with a width of $90 \mathrm{~km} / \mathrm{s}$ is $40 \%$ and with a width of $500 \mathrm{~km} / \mathrm{s}$ is $60 \%$, leading to an average velocity of $\sim 540 \mathrm{~km} / \mathrm{s}$.

In Figure 6, we show the $z$ distribution above the Galactic disk for the detected pulsars (shaded histogram) and for the simulated pulsars (regular histogram). Under the assumptions of the model, the predicted distribution is a little wider that the one for detected pulsars, having scale heights of $152 \mathrm{pc}$ and $182 \mathrm{pc}$, respectively. We realize that many assumptions in our model are interrelated and decreasing the overall radio luminosity of the ACC model leads to a difference in the $z$ distribution as pulsars are radio dimmer and must be closer to be detected. We have chosen to keep the velocity model and overall radio luminosity model of ACC making as few necessary changes to the ACC model as needed.

\subsection{Reduction of the radio luminosity}

Using the radio luminosity of $A C C$, we find that the simulated radio pulsars are too bright, with too many distant pulsars being detected and predicting a neutron star birth rate of 0.11 per century, with no $\gamma$-ray pulsars predicted to be detected by EGRET. In ACC all the pulsar surveys used in the study were at frequency near $400 \mathrm{MHz}$. In the set of surveys chosen for this study there are two groups with one having frequencies near 400 $\mathrm{MHz}$ and the other group with frequencies near $1400 \mathrm{MHz}$. Since the PMBPS $S_{\min }$ is best accounted for in our simulation and this survey at $1374 \mathrm{MHz}$ detected most of the pulsars observed in the Jodrell Bank 2 survey at $1400 \mathrm{MHz}$ and the Parkes 1 survey at $1520 \mathrm{MHz}$, we selected only the PMBPS pulsars to represent the high frequency (HF) surveys, while the other selected surveys in our study near $400 \mathrm{MHz}$ represent the low frequency (LF) surveys. Focusing primarily on the distributions of the pulsar distance, DM and flux at $400 \mathrm{MHz}$ and $1400 \mathrm{MHz}$, we first set the spectral indices (preserving 0.5 between core and cone indices) to give the same birth rate for these two frequency groups, then we set the over all luminosity to given a reasonable birth rate of $\sim 1.5$ neutron stars per century. We obtain the same birth rate for each group with spectral indices of $\alpha_{\text {core }}=-2.1$ and $\alpha_{\text {cone }}=-1.6$. These spectral indices describe the primary spectra of the radio pulsars before the selection effects of the 
characteristics of the chosen radio surveys.

In our simulation, we calculate the fluxes at the frequency of each of the surveys in our selected group as well as at 400 and $1400 \mathrm{MHz}$ by averaging the pulse profile for a given random viewing geometry for each pulsars. From the calculated S400 and S1400, we obtain a spectral index for each pulsar. In the simulation with all the surveys, we simulated 9780 (ten times the number in our select group of surveys to improve statistics) radio pulsars as detected by these surveys and find an average spectral index of -1.8 and a standard deviation of 0.2. Lorimer et al. (1995) measured spectral indices of 280 pulsars by measuring fluxes at radio frequencies between 408 and $1606 \mathrm{MHz}$ with the distribution having a dependence on the characteristic age of the pulsar with a dependence, $\alpha=-1.7+0.2 \log (\dot{P} / P)$, with a standard deviation of 0.6 with respect to this dependence. Maron et al. (2000) extended to lower and higher frequencies the study of Lorimer et al. (1995) and obtained an average spectral index of -1.8 with a standard deviation of 0.2 .

To show the overall effect of the reduction of the radio luminosity used in ACC, we show in Figure 7 the comparisons of the distributions for the pulsar distance, flux at $1400 \mathrm{MHz}$ and DM for 620 pulsars detected with only the PMBPS and an equal number simulated pulsars for this survey alone. Given that we are calculating the $S_{\min }$ for the PMBPS according to the formulation used in Manchester et al. (2001), we believe that the $S_{\min }$ for this survey is the best described in the group of our selected surveys, and with all pulsars detected at $\sim 1400$ $\mathrm{MHz}$ sky temperature effects are minimized, reducing further uncertainties. Indicated in Figure 7 are the factors, $f_{\text {red }}$, used to reduce the radio luminosity of the ACC model and the resulting distributions. Since the ACC model studied pulsar surveys at $400 \mathrm{MHz}$, the factor $f_{\text {red }}$ represents a reduction of the ACC $400 \mathrm{MHz}$ luminosity by this factor. As the radio luminosity is reduced, the comparison of the distances and DMs improves, but disagreement increases between the distribution of the simulated and detected radio flux at $1400 \mathrm{MHz}$. There are significantly more pulsars simulated with lower radio fluxes than those detected by this survey, suggesting that perhaps certain aspects of the emission geometry are still not adequately described. We find that the shape of the flux distribution is not very sensitive to the features of the conal geometry, such as the radius and width. We also wanted to predict a reasonable neutron star birth rate per century, which varies from 0.6 at $f_{\text {red }}=20$ to 1.6 at $f_{\text {red }}=80$. We chose to reduce the radio luminosity by a factor of $f_{\text {red }}=60$ in subsequent simulations in the following figures compromising between good agreement of the distances and DMs and less desirable agreement of the fluxes. These factors predict a birth rate of 1.38 neutron stars per century for the case of all nine radio surveys. 


\section{Results}

In Figure 8, we present the distribution of our select group of 978 detected pulsars in the ATNF pulsar catalog (8a) and the same number of simulated pulsars (8b) as a function of period derivative and period. The solid lines represent constant dipole magnetic field of $10^{11-14} \mathrm{G}$, and the dashed lines correspond to the curvature and nonresonant inverse Compton scattering death lines. As dotted curves, we show paths in the diagram for 4 pulsars assuming a field-decay constant of $2.8 \mathrm{Myr}$, all with ages of $10^{7}$ years, and with the indicated initial magnetic fields in $B_{12}$ units. We show the age lines assuming field decay as dot-dashed lines. Due to field decay, these lines are very different from the characteristic age lines, with the oldest pulsars being $10 \mathrm{Myr}$ rather than $1 \mathrm{Gyr}$ assuming no field decay. As indicated in Table 1, we chose to represent the initial magnetic field distribution with two Gaussians. The initial magnetic fields of the four evolutionary paths portrayed in Figure 8 were chosen to represent the means of the two Gaussians, the higher mean plus the high field component's width and the lower mean minus the lower field component's width. As a result, most of the simulated pulsars will lie within the lowest and highest paths. Due to a $2.8 \mathrm{Myr}$ decay constant, the knee-like, curved portions in these paths begins before $1 \mathrm{Myr}$ and become vertical after a few Myr. The paucity of pulsars in the two regions indicated with the circle and LB and HB for low and high field (Figure 8a) can be explained in terms of decay of the surface magnetic field on a time scale of $2.8 \mathrm{Myr}$. While it may be true that one can tailor a radio luminosity law to account for the observed distribution, field decay leads naturally to the funnel-shaped distribution of the detected pulsars.

In Figure 9, we present the comparisons between detected and simulated statistics for the indicated parameters. The shaded histograms represent the distributions of the detected pulsars while the regular histograms correspond to the model simulations. The model simulation over-predicts the number of pulsars with short periods, large period derivatives and larger distances. Improved comparisons can be obtained by making the radio luminosity not as strongly dependent on the period by decreasing the exponent from -1.3 to -0.9 and that of the period derivative from 0.4 to 0.3 . The main discrepancy lies in the comparisons of the distance distributions and flux distributions (Figure 5). The agreement in the flux distributions for only the PMBPS, shown back in Figure 5, is better with a reduction factor of $f_{\text {red }}=20$ making the pulsars more radio bright. However, the agreement between the distance distributions is significantly worse and the birth rate is too low. Perhaps the inability to find agreement in the radio flux and distance distribution may stem from our underlying assumption that radio pulsars are standard candles or may be indicating that spiral arm structure may have to be used for the birth location of neutron stars in order to improve the agreement between the simulated and observed distributions. 
In Figure 10, we show the distributions in Galactic coordinates as Aitoff projections for 978 pulsars detected (10a) and simulated (10b). The strong contribution to the Galactic disk is due primarily to the PMBPS, adding nearly half of the total number of pulsars that are unique to this survey.

We also simulate the $\gamma$-ray pulsar detections by EGRET, AGILE and GLAST, using the assumptions discussed earlier, and independently simulate detection of radio pulsars. However, the $\gamma$-ray pulsars are flagged as radio-loud if their fluxes are higher than the minimum sensitivities of the select group of surveys; otherwise they are radio-quiet. Therefore, we can predict the number of radio-loud and radio-quiet $\gamma$-ray pulsars detected as point sources by each of the three instruments. For known radio pulsars, with measured periods and period derivatives, the $\gamma$-ray instrument can detect them as point sources and obtain a pulsed detection through reliable epoch folding. GLAST will have the sensitivity and ability to perform blind period searches and detect pulsation without a radio ephemeris. Table 2 indicates the simulated pulsar statistics for the radio-quiet and radio-loud $\gamma$-ray pulsars as detected by the three instruments.

Table 2

\begin{tabular}{c|c|c|c|c}
\hline & \multicolumn{4}{|c}{ Simulated Pulsar Statistics } \\
\hline \hline & \multicolumn{2}{|c|}{$\begin{array}{c}\text { All Nine Surveys } \\
\text { (978 radio pulsars) }\end{array}$} & $\begin{array}{r}\text { All Surveys Excluding PMBPS } \\
\text { (546 radio pulsars) }\end{array}$ \\
\hline Birth Rate & \multicolumn{2}{|c|}{1.38} & \multicolumn{2}{c}{1.46} \\
\hline & Radio Quiet & radio-loud & radio-quiet & radio-loud \\
\hline EGRET & 7 & 19 & 10 & 15 \\
AGILE & 13 & 37 & 19 & 36 \\
GLAST & 276 & 344 & 436 & 209 \\
\hline
\end{tabular}

To improve the statistics, we run the simulation until the number of radio pulsars detected by the chosen set of radio surveys is equal to ten times the number of actual detected radio pulsars by those surveys. We then renormalize by dividing by a factor of ten. EGRET observed all of the radio pulsars that were detected by all the radio surveys excluding the PMBPS, out of which EGRET detected $8 \gamma$-ray pulsars including Vela, the Crab, B1951+32, $\mathrm{B} 1706-44$, B1055-52, B0656+14, J1048-5832, and the radio-quiet $\gamma$-ray pulsar, Geminga plus a couple of other candidate pulsars bring the total to perhaps 12 (Kanbach 2003). Excluding the PMBPS, our simulation predicts that EGRET should have seen 15 radio-loud and 10 radio-quiet $\gamma$-ray pulsars and a neutron star birth rate of 1.46 per century. With all nine surveys, the predicted birth rate of 1.36 is $5 \%$ smaller and, therefore, the total number of $\gamma$ ray pulsars has also dropped by $5 \%$ (GLAST) within statistics. The EGRET Third catalogue 
(Hartman et al. 1999) contains 170 unidentified point sources, some of which are expected to be pulsars. Sensitive searches performed with Chandra and the Parkes multi-beam telescopes have resulted in a few new pulsars within the error boxes of the unidentified EGRET sources (Halpern et al 2001; D'Amico et al. 2001). Correlating the positions of the radio pulsars detected in the PMBPS with the EGRET unidentified sources, Torres et al. (2001) found 14 positional coincidences. With the nearly completed PMBPS, Kramer et al. (2003) found about 38 positional coincidences, and they determined that $19 \pm 6$ are statistically likely to be real associations. So it would seem then that adding the PMBPS should convert radioquiet $\gamma$-ray pulsars into radio-loud $\gamma$-ray pulsars as detected by EGRET. This is clearly the case for GLAST and there is a significant conversion of radio-quiet to radio-loud pulsars for AGILE and EGRET when the PMBPS is added. Since our present simulation overpredicts the number of short period, energetic pulsars, mostly detected by the eight surveys (without PMBPS) prior to EGRET, our results are over-predicting the number of radioloud pulsars detected by EGRET from these surveys. However, the number of radio-loud pulsars predicted for all surveys including PMBPS is consistent with the number of plausible coincidences found by Kramer et al. (2003).

In Figure 11, we present the positions in the $\dot{P}-P$ diagram of the known $\gamma$-ray pulsars detected by EGRET (10a) and of those simulated for EGRET (10b), AGILE (10c) and GLAST (10d) where radio-loud $\gamma$-ray pulsars are shown as solid circles and radio-quiet $\gamma$-ray pulsars are shown as crosses. Younger pulsars have higher $\gamma$-ray luminosities that decrease as they approach the curvature death line where curvature radiation $\gamma$-rays can no longer produce electron-positron pairs. The $\gamma$-ray luminosity decreases significantly for older pulsars below the curvature radiation death line (Harding et al. 2002), where the main mechanism of pair production is via inverse Compton scattering of the thermal soft Xrays from the stellar surface. Pulsars below the nonresonant Compton scattering death line are unable to produce pairs and become radio-quiet. Our simulations predict that GLAST will detect 276 radio-quiet and 344 radio-loud $\gamma$-ray pulsars; a significant improvement over EGRET. With GLAST ability to perform blind period searches, we predict that out of the 276 radio-quiet $\gamma$-ray pulsars, 17 pulsars will be detected as pulsed sources. AGILE, scheduled to launch before GLAST, should detect 13 radio-quiet and 37 radio-loud $\gamma$-ray pulsars using the nominal sensitivity map. The best and worse case-maps predict 24 and 9 radio-quiet and 64 and 27 radio-loud $\gamma$-ray pulsars, respectively. As all-sky sensitivity and threshold maps are improved, these numbers will vary somewhat.

We present in Figure 12 the Aitoff projections of the $\gamma$-ray pulsars observed by EGRET and those simulated for EGRET, AGILE and GLAST. Most of the pulsars detected by GLAST will be young pulsars with ages $\sim 10^{5}$ years and within $500 \mathrm{pc}$ of the Galactic disk. The asymmetric distribution of radio-loud and radio-quiet $\gamma$-ray pulsars is a result of the 
PMBPS detecting radio pulsars from $\ell=260^{\circ}$ to $\ell=50^{\circ}$, mostly on the right side of the figure.

In order to further explore the effect of the parameterization of the beam geometries of the radio and $\gamma$-ray emission, one has to study the pulse profiles and the correlation between the radio profile and the $\gamma$-ray profile. In Figure 13, we present a select pair of examples of simulated radio and $\gamma$-ray profiles for two radio-loud pulsars "detected" by EGRET with periods of $528 \mathrm{~ms}(13 \mathrm{a})$ and $41 \mathrm{~ms}(13 \mathrm{~b})$. The two pulsars also have similar impact angles of $4.1^{\circ}(13 \mathrm{a})$ and $-5.5^{\circ}(13 \mathrm{~b})$. The radio profiles presented here are for $400 \mathrm{MHz}$ and the $\gamma$-ray profiles are for greater than $100 \mathrm{MeV}$. The ACC model predicts ratios of the radio core-tocone flux of 13 (Figure 13a) and 163 (Figure 13b), suggesting that the conal contribution to the flux is minor. However, the viewing geometry is important also. The pulse profile in the line-of-sight intersects the radio cone and the outer portion of the radio core, displaying two radio peaks, but only one curvature radiation $\gamma$-ray peak. Due to the large impact angle, the radio core does not significantly contribute to the overall profile as this is a fairly long period pulsar. The other pulsar in Figure $13 \mathrm{~b}$ has a similar impact parameter but with a shorter period, therefore the radio core clearly dominates the profile and the two conal peaks from the $\gamma$-ray pulse are manifested. Since in this model the $\gamma$-ray emission originates within 2.5 stellar radii of the surface and the radio core emission is believed to come from similar altitudes above the stellar surface, there would be little aberration or time delay between them and they should appear correlated with the radio core peak being in phase with the single $\gamma$-ray peak, as in Figure 13a, or being in between the two $\gamma$-ray peaks, as in Figure $13 \mathrm{~b}$. On the other hand the radio cone beam is believed to arise from a higher altitude region and the effects of aberration and time delay, not included in our model, might shift the peaks to earlier phase relative to the $\gamma$-ray or radio core in the pulse profile. This effect is actually observed in a number of triple radio profiles, where the core component lags the center of the cone (Gangadhara \& Gupta 2001). In the ACC model with core dominated short-period pulsars, we find that $54 \%$ of the EGRET radio-loud $\gamma$-ray pulsars have two $\gamma$-ray peaks in the pulse profile and are all core dominated, exhibiting a single (core) radio peak in the profile. The other $46 \%$ have a single $\gamma$-ray peak with a variety of radio profiles, including a single core peak, core and conal peaks, and two conal peaks. However, the fact that all two-peaked $\gamma$-ray profiles are core dominated is contrary to the few EGRET $\gamma$-ray pulsars, typically consisting of two $\gamma$-ray peaks with a single radio peak leading the $\gamma$-ray peaks (Thompson et al. 1997). This significant discrepancy between the observed and simulated radio and $\gamma$-ray profile correlations either questions the parameterization of the core to cone radio flux in the ACC model suggesting that these short period pulsars are different, having their profiles dominated by cone emission, or questions the polar cap $\gamma$-ray emission model we have used. 
By contrast, several recently discovered, young X-ray pulsars show single broad peaks in their profiles and also single radio peaks in phase with the X-ray peaks (Lorimer 2003 and references therein). They also have very low radio flux and luminosity. Harding et al. (2003) have shown that the radio characteristics can be understood in the ACC model if we are viewing the edge of the cone beams at large impact parameter, so that the cone beam appears single and the core component is not seen. In the polar cap model emission geometry, the high-energy emission peak would be in phase with the single radio peak, as observed. A number of these are coincident with EGRET sources and AGILE may measure the $\gamma$-ray profiles.

\section{Discussion}

We have included radio and $\gamma$-ray beam geometries into our Monte Carlo code that simulates population statistics for radio and $\gamma$-ray pulsars, making predictions for the number of radio-quiet and radio-loud $\gamma$-ray pulsars detected by the instruments: EGRET, AGILE and GLAST. The radio beam geometry is tailored after the phenomenological model of ACC with slight modifications to include the radius-to-frequency mapping of Mitra \& Deshpande (1999). In the ACC model, radio pulsars are assumed to be standard candles with their radio luminosity described by a simple power law in period and period derivative. The $\gamma$-ray beam geometry has been derived from the theoretical work of Muslimov \& Harding (2003) describing the emission from the slot gap in the polar cap model. These enhancements are significant improvements over our previous studies of Gonthier et al. (2002). We have added the PMBPS to our select group of radio surveys, we have used the new distance model of Cordes \& Lazio (2003), we have added all-sky threshold maps for EGRET (Grenier private communication) and AGILE (Pellizzoni private communication), and we have used realistic $S_{\min }$ thresholds for the PMBPS (Crawford private communication). Neutron stars are assumed to be born with a constant birth rate, with a Galactic distribution described by Paczyński (1990) with a flat distributions in their initial period and a Gaussian distribution in their magnetic field, and their trajectories are evolved to the present within the Galactic potential (Paczyński 1990). We find better agreement when the magnetic field is assumed to decay exponentially with a decay constant of $2.8 \mathrm{Myr}$, which is somewhat shorter than the decay constant in Gonthier et al. (2002) that assumed an entirely different beam geometry and radio and $\gamma$-ray luminosity models.

In order to obtain agreement between observed and simulated radio pulsar distance distributions, a reasonable birth rate of 1.45 neutron stars per century and a reasonable number of $\gamma$-ray pulsars observed by EGRET, we find it necessary to significantly reduce 
by a factor 60 the overall radio luminosity of ACC model. The major problem with our radio emission model is that we are not able to simultaneously fit the distance distribution and the radio flux distribution as seen in Figure 5. We found that slight adjustments to the radio beam geometry do not affect these distributions. This inadequacy perhaps challenges some major assumptions of the model such as that radio pulsars are standard candles or that short period pulsars are core dominated. There is a lack of theoretical insight into the mechanism for the radio luminosity. Randomizing the radio luminosity about the expected value does indeed achieve some improvement in the flux distribution. The disagreement between the inferred and the simulated distance distributions may be a result of assuming a smooth distribution of neutron stars in the Galactic plane, rather than taking into account the spiral structure of the Galaxy. It is very apparent that.most pulsars are detected in spiral arm regions.

Our simulation is normalized to the number of radio pulsars observed by the selected group of surveys and, therefore, a neutron star birth rate is predicted, as well as the number of $\gamma$-ray pulsars observed by each instrument as shown in Table 2 . We expected by adding the PMBPS that the ratio of radio-quiet $\gamma$-ray pulsars to radio-loud $\gamma$-ray pulsars would decrease for EGRET given the positional coincidences found between the Parkes multi-beam radio pulsars within EGRET error boxes. Recently Kramer et al. (2003) estimated about $19 \pm 6$ associations, which suggests about 20 radio-quiet $\gamma$-ray pulsars detected by EGRET that become radio-loud when the PMBPS is included. The simulated ratio of radio-loud to radioquiet $\gamma$-ray pulsars decreases by $45 \%$ from 2.7 (with PMBPS) to 1.5 (without PMBPS) for EGRET. For AGILE there is also a similar decrease of $34 \%$ from 2.9 (with PMBPS) to 1.9 (without PMBPS), but very evident for GLAST as the ratio decreases significantly by $62 \%$ from 1.25 (with PMBPS) to 0.5 (without PMBPS).

Our model assumes that pulsars are evenly distributed azimuthally throughout the Galactic disk peaking radially at $4.5 \mathrm{kpc}$ and falling off exponentially with distance from the center. Our model does not assume the spiral structure of the Galaxy where the pulsars are indeed born. The location of most of the detected pulsars is clearly correlated with the spiral arms of the Milky Way. Perhaps the difficultly of our model in reproducing the distance and flux distributions is a result of the lack of including this strong correlation of the location of pulsars and the spiral arms.

We believe that the fact that we cannot reproduce the decrease in the ratio of radioloud to radio-quiet $\gamma$-ray pulsars for EGRET points to a significant limitation of our overall model. The fact that we are not able to simultaneously account for the detected radio flux and inferred distance distributions and that the radio pulse profiles do not correlate with the $\gamma$-ray profiles as those detected by the EGRET, also suggest that we are not adequately 
accounting for everything. The problem with the pulse profiles perhaps implies that the parameterization of the ratio of radio core to cone with period in the ACC model does not apply for $\gamma$-ray pulsars. Recently Crawford, Manchester \& Kaspi (2001) reported that six young pulsars have large linearly polarized profiles characteristic of conal emission. Yet in the ACC model, young pulsars with short periods would be characterized by significant core emission. In order to avoid the core component, the impact angle must be fairly large to observe a single peak from the edge of a conal beam. The pulsar J1105-6107 has a period of $0.063 \mathrm{~s}$, and the ACC model predicts an integrated flux core to cone ratio of about 60 making it difficult to avoid seeing the core beam when a significant mean conal flux of 1.0 $\mathrm{mJy}$ is detected. Perhaps partial conal emission becomes dominant for short period pulsars as suggested by Manchester (1996).

The correlation between the radio and $\gamma$-ray beam profiles and the shapes of the profiles are sensitive to the geometry of the beams as well as the viewing geometry. In the ACC model that we have used in our simulations, short period pulsars have fluxes that are core dominated. All two-peaked $\gamma$-ray pulse profiles display a single radio core peak in the radio pulse profile. In order to see a cone dominated radio profile, the impact parameter must be so large that single-peaked $\gamma$-ray profiles are seen. This appears contrary to the features of the most of the $\gamma$-ray profiles detected EGRET and again raises the issue of whether short period pulsars are core dominated and perhaps are really partial cone dominated as suggested by Manchester (1996) and, more recently, by Crawford, Manchester \& Kaspi (2001).

We conclude that a better radio beam model is required to account for the observed characteristics of radio pulsars. In addition, recent theoretical work by Dyks \& Rudak (2003) and Muslimov \& Harding (2004) indicates the importance of including the caustic component of $\gamma$-rays in the emission geometry. We hope that in the near future, we will be able to include more realistic emission geometries of the radio and $\gamma$-ray beams.

\section{Acknowledgements}

We would like to acknowledge the many conversations with Zaven Arzoumanian and the insight that he has given to us. We also thank an anonymous referee for suggesting many improvements to the manuscript. We appreciate the $S_{\min }$ code for the Parkes multi-beam pulsar survey from Froney Crawford. We are grateful to Alberto Pellizzoni in providing us with the all-sky sensitivity maps for AGILE, as well as Isabelle Grenier in allowing us to use their EGRET all-sky sensitivity map. We express our gratitude for the generous support of Research Corporation (CC5813), of the National Science Foundation (REU and AST-0307365), and NASA Astrophysics Theory Program. 


\section{REFERENCES}

Arons, J., \& Scharlemann, E.T. 1979, ApJ, 231, 854

Arzoumanian, Z., Chernoff, D.F., \& Cordes, J.M. 2001, ApJ, 568, 289

Chandler, A. M. et al. 2001, ApJ, 556, 59

Cheng, K.S. et al. 2000, ApJ, 537, 964

Clifton, T.R. \& Lyne, A.G. 1986, Nature, 320, 43

Cordes, J.M., \& Lazio, T.J.W. 2002, astro-ph/0301598

Crawford, F., Manchester, R.N., \& Kaspi, V.M. 2001, AJ, 122, 2001

D'Amico, N. et al. 2001, ApJ, 552, 45

Daugherty, J. K., \& Harding A. K. 1996, ApJ, 458, 278

Dewey, R.J., Taylor, J.H., Weisberg, J.M., \& Stokes, G.H. 1985, ApJ, 294, L25

Dyks, J., \& Rudak, B. 2003, astro-ph/0303006

Gavriil, F.P. et al. 2003, American Astronomical Society, HEAD meeting 35

Gonthier, P.L. et al. 2002, ApJ, 565, 482

Gangadhara, R. T. \& Gupta, Y. 2001, ApJ, 555, 31

Halpern, J.P. et al. 2001, ApJ, 547, 323

Hansen, B.M.S. \& Phinney, E.S. 1997, MNRAS, 291, 569

Harding, A. K. 2001, in AIP Conf. Proc. 558, High Energy $\gamma$-Ray Astronomy, ed. by F. A. Aharonian and H. J. Völk. (New York: AIP), 115

Harding, A. K., Arzoumanian, Z., Gonthier, P. L. \& Cordes, J. M. 2003, BAAS, 35, 633

Harding, A. K. \& Daugherty, J. K. 1999, A\&AS, 120, 10

Harding, A.K., Muslimov, A.G., \& Zhang, B. 2002, ApJ, 576, 366

Hartman, J.W. et al. 1997, A\&A, 322, 477

Hartman, R. et al. 1999, ApJ Supp., 123, 79

Kanbach, G. 2000, American Astronomical Society, HEAD Meeting 32, Bulletin of the American Astronomical Society, 1206

Kramer, M. et al. 2003, MNRAS, 342, 1299

Lorimer, D.R. et al. 1995, MNRAS, 289, 592

Lorimer, D.R., Bailes, M., \& Harrison, P.A. 1997, MNRAS, 289, 592 
Lorimer, D.R. 2003, Conf. Proc., "Pulsars, AXPs, and SGRs observed with Bepposax and other observatories", Marsala, Italy, in preparation

Lyne, A.G., \& Lorimer, D.R. 1994, Nature, 369, 127

Manchester, R.N. et al. 1996, MNRAS, 279, 1235

Manchester, R.N. et al. 2001 MNRAS, 328, 17

Maron, O. et al. 2000, A\&AS, 147, 195

Mattox, J. et al. 1996, A \& AS, 120, C95

McLaughlin, M. \& Cordes, J. M. 2000, ApJ, 538, 818

Mitra, D., \& Deshpande, A.A. 1999, A\&A, 346, 906

Morris et al. 2002 MNRAS, 335, 275

Muslimov, A.G., \& Harding, A.K. 2003, ApJ, 588, 430

Muslimov, A.G., \& Harding, A.K. 2004, ApJ, in press

Narayan, R., \& Ostriker, J.P. 1990, ApJ, 352, 222

Paczyński, B. 1990, ApJ, 348, 485

Romani, R. W., \& Yadigaroglu, I.-A. 1995, ApJ, 438, 314

Shapiro, S. L. \& Teukolsky, S. A. 1983, Black Holes, White Dwarfs, and Neutron Stars The Physics of Compact Objects (John Wiley \& Sons New York), 278

Taylor, J. H., Manchester, R. N., \& Lyne, A. G. 1993, ApJS, 88, 529

Thompson, D.J. 2001 in AIP Conf. Proc. 558, High Energy $\gamma$-Ray Astronomy, ed. by F. A. Aharonian and H. J. Völk. (New York: AIP), 103

Thompson, D. J., Harding, A.K., Hermsen, W., \& Ulmer, M. 1997, in Proc. of 4th Compton Symposium, ed. C.D. Dermer, M.S. Strickman \& J. Kurfess, p. 39

Torres, D.F. et al. 2001, ApJ, 560, L155

Zhang, B., \& Harding, A. K. 2000, ApJ, 532, 1150 


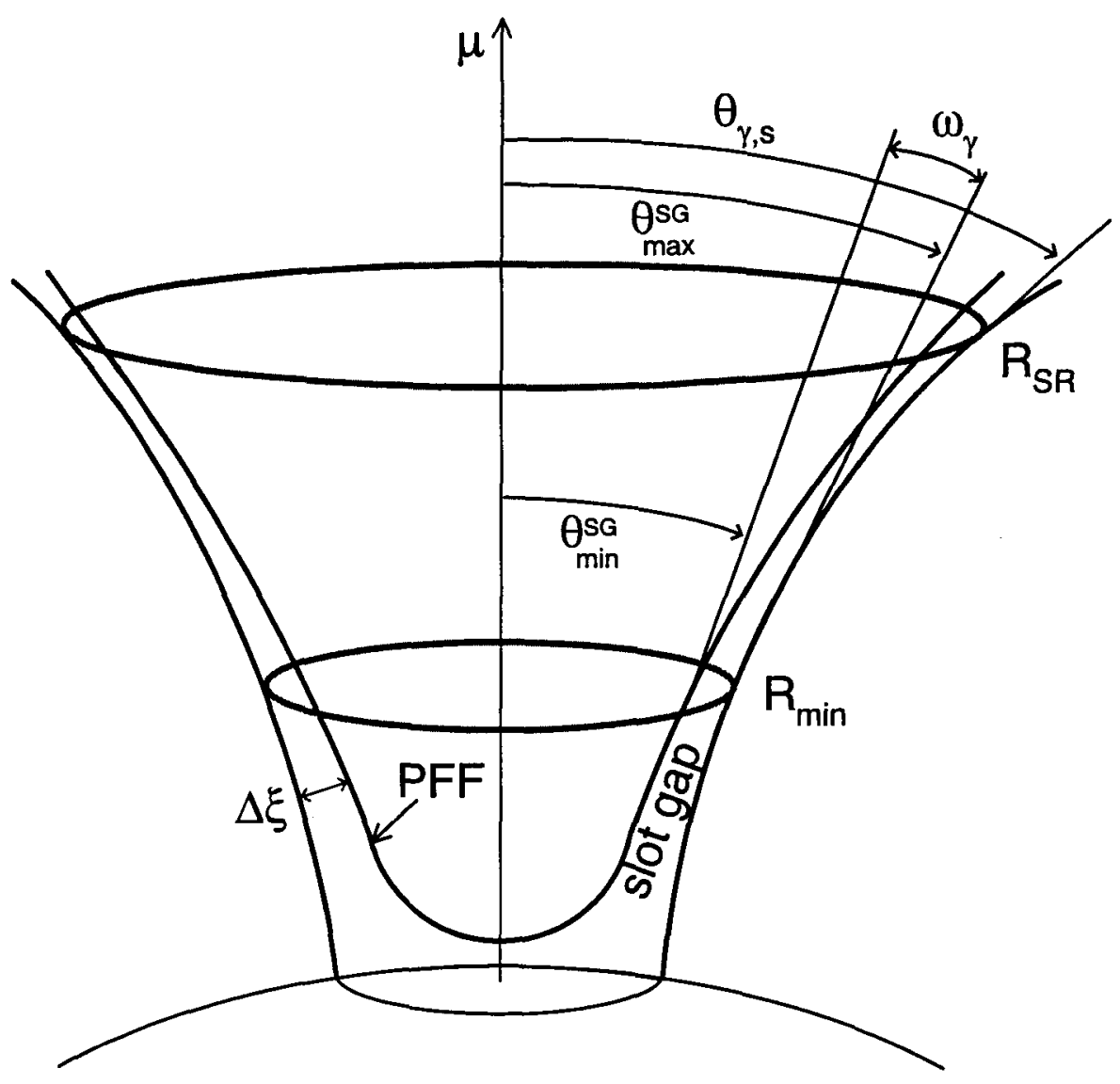

Fig. 1.- Geometry of the slot gap emission relative to the magnetic moment vector, $\mu$. The pair formation front (PFF) curves upward near the last open field line and forms the inner boundary of the slot gap with width $\Delta \xi$ (in units of polar cap half-angle). $R_{\min }$ and $R_{S R}$ are the minimum and maximum radii of pair synchrotron radiation. $\theta_{\min }^{S G}$ and $\theta_{\max }^{S G}$ are the tangent angles to field lines at the inner and outer edge of the slot gap at $R_{\min }$. 


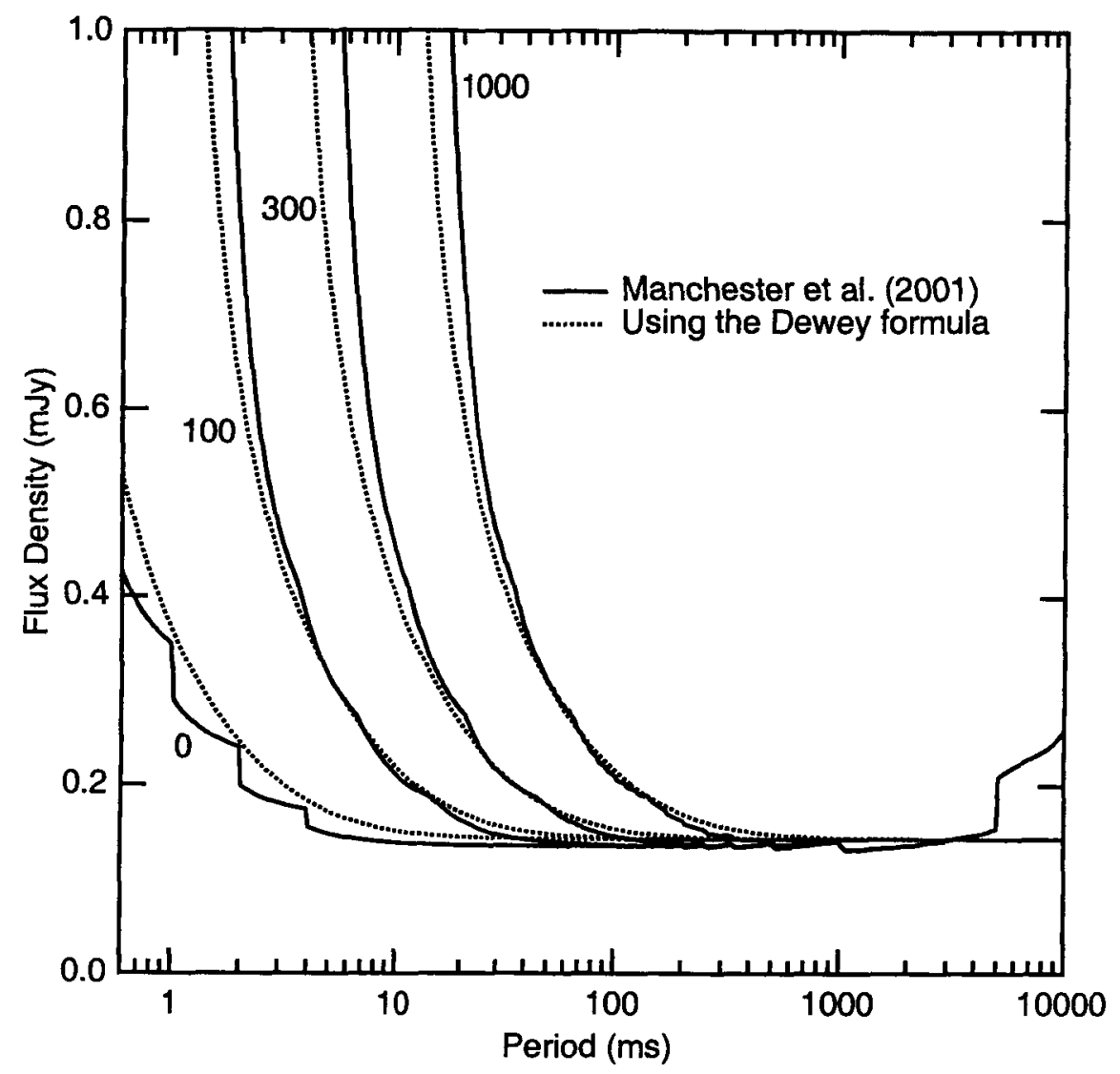

Fig. 2.- The minimum radio flux, $S_{\min }$, detectable by the Parkes multi-beam pulsar survey for the indicated DM (in $\mathrm{pc} \mathrm{cm}^{-3}$ ) as a function of period, calculated as in Manchester et al. (2001) (solid curves) and calculated using the formula in Dewey et al. (1985) (dotted curves) with an additional factor of 2 (see text). 


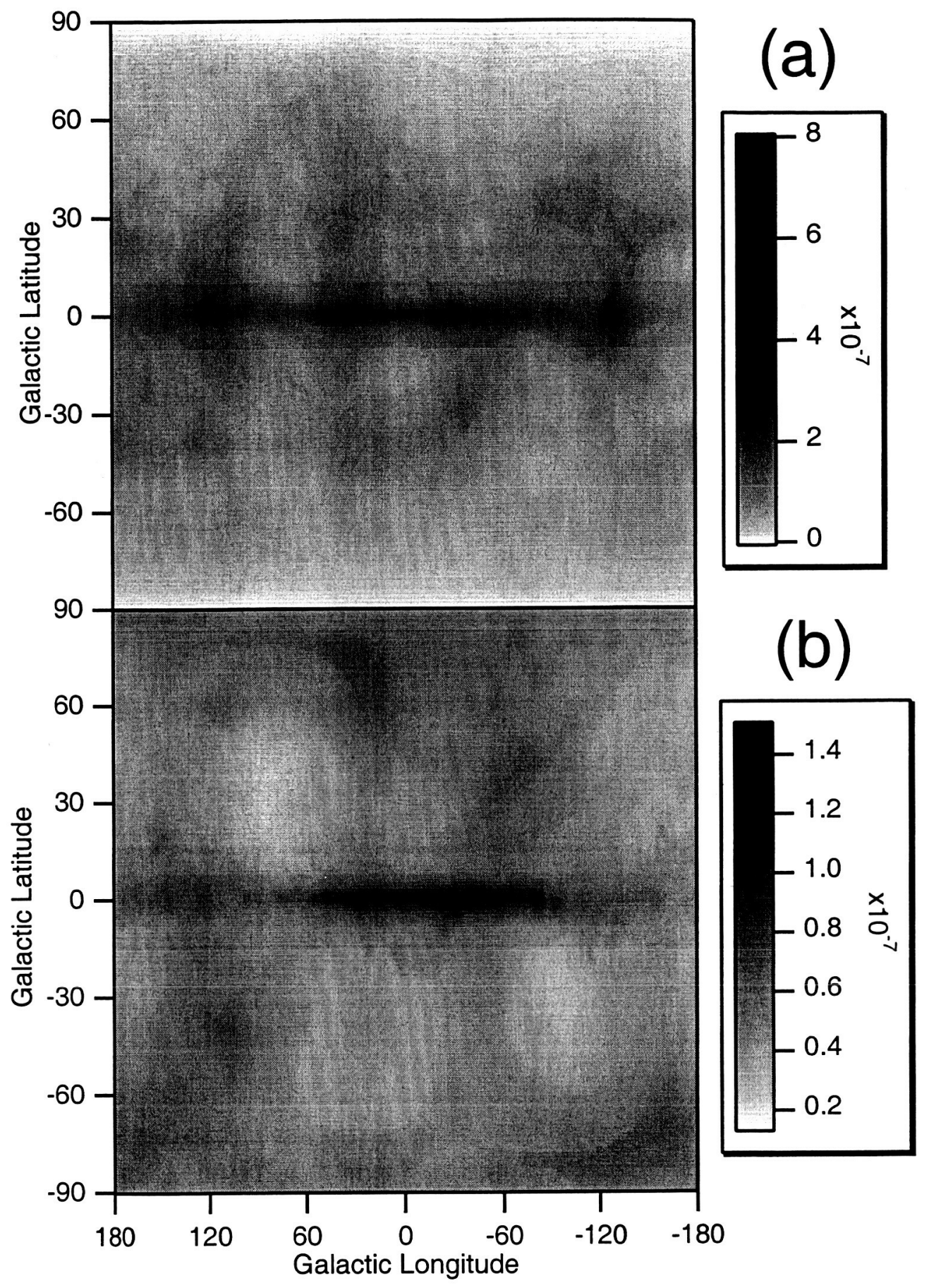

Fig. 3.- Gamma-ray flux threshold maps in Galactic latitude versus longitude for EGRET (I. Grenier, private communication) in (a) and for AGILE (A. Pellizzoni, private communication) in (b) for the nominal case. Gray scale is in units of photons $\mathrm{s}^{-1} \mathrm{~cm}^{-2}$. 


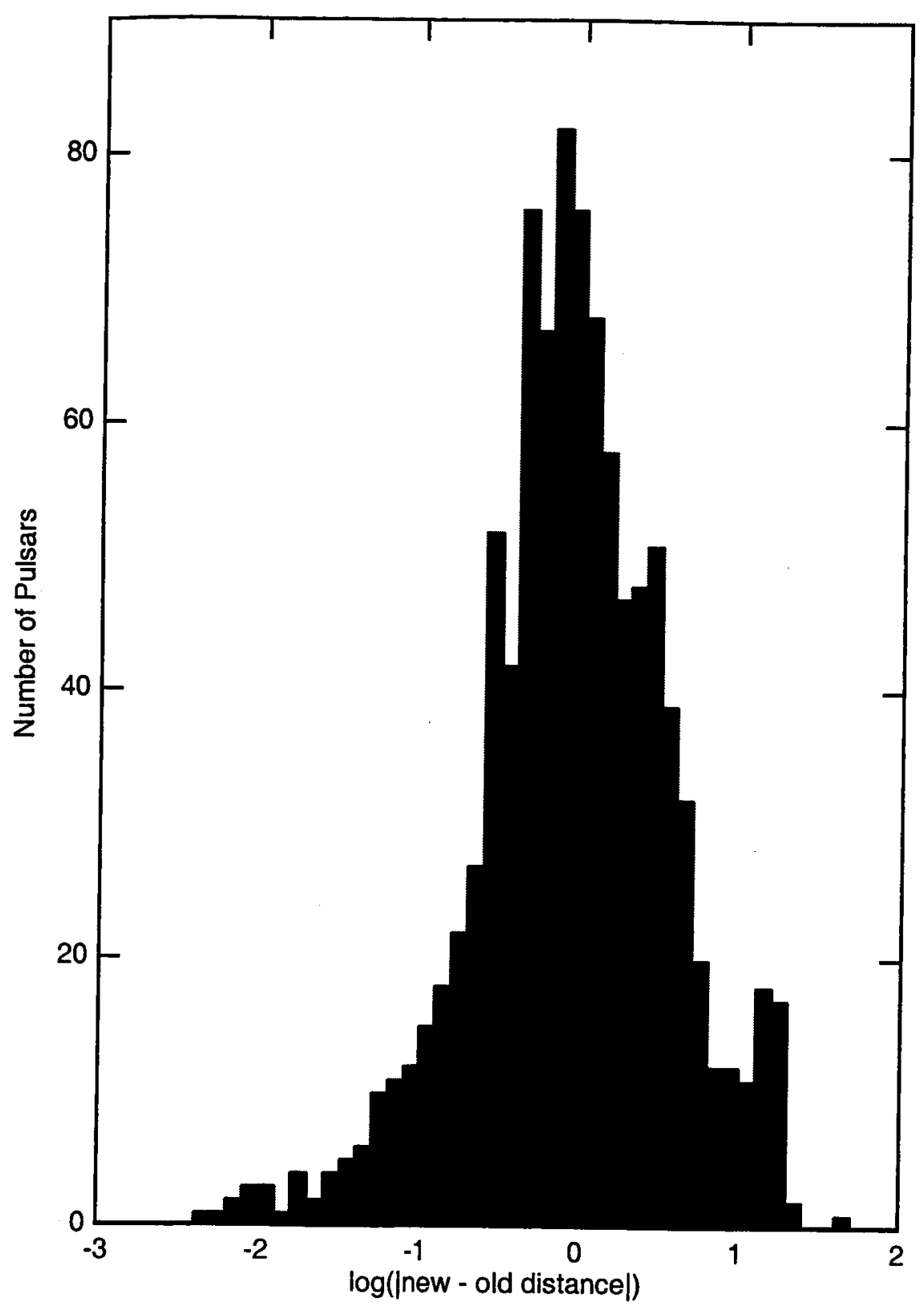

Fig. 4.- The distribution of the logarithm of absolute value of the difference between the distance of pulsars obtained from the new (Cordes \& Lazio 2002) and old (Taylor \& Cordes 1993) electron density models. 


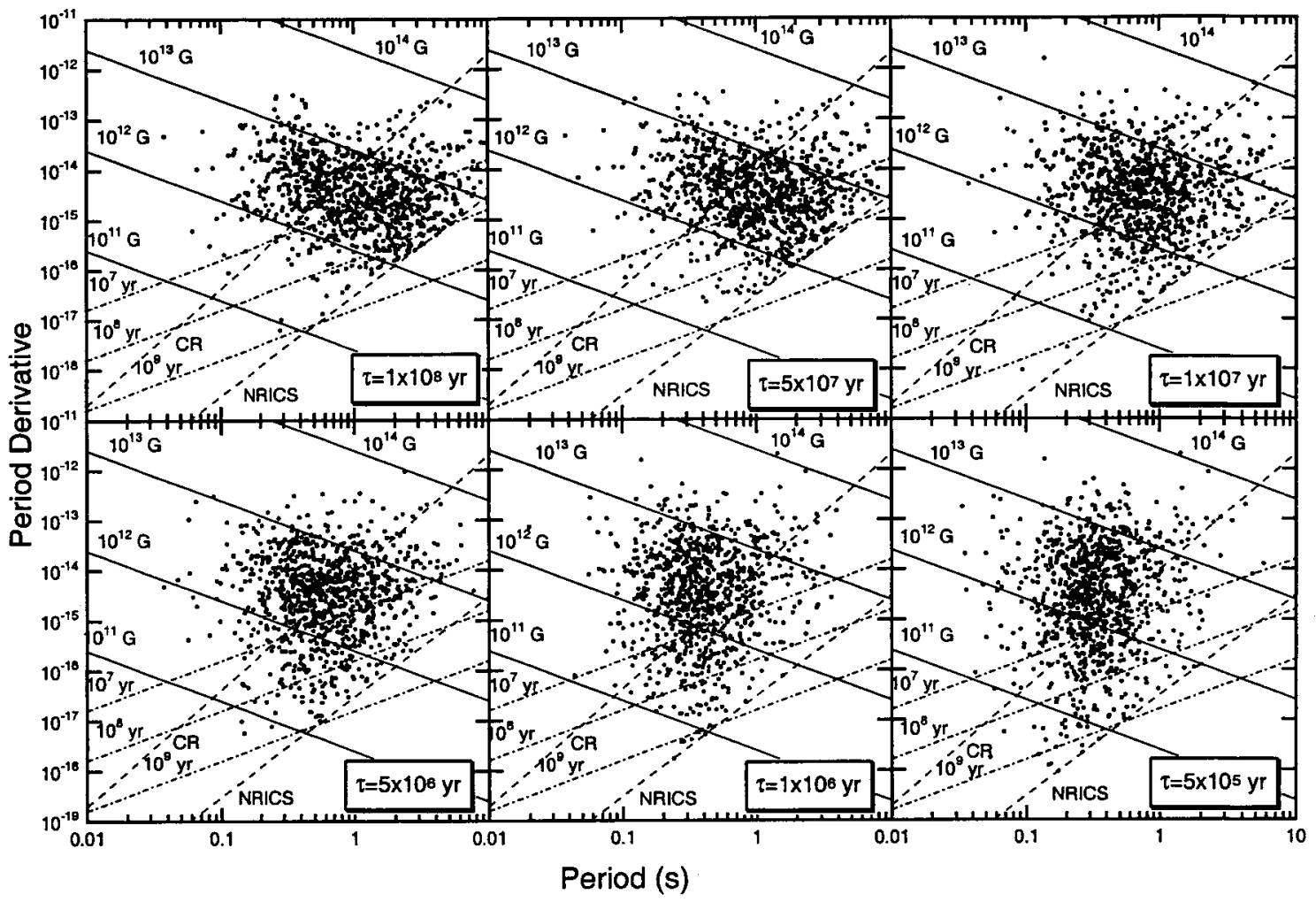

Fig. 5.- Distributions of simulated radio pulsars as a function of the period derivative and period for the indicated decay constants of the magnetic field. Dashed lines represent the death lines for curvature radiation (upper) and for nonresonant inverse Compton scattering (lower). Dot-dashed lines represent the indicated pulsar age (in yr) and solid lines represent the indicated magnetic surface field strength (in units of $G$ ) assuming a constant dipole spin-down field. 


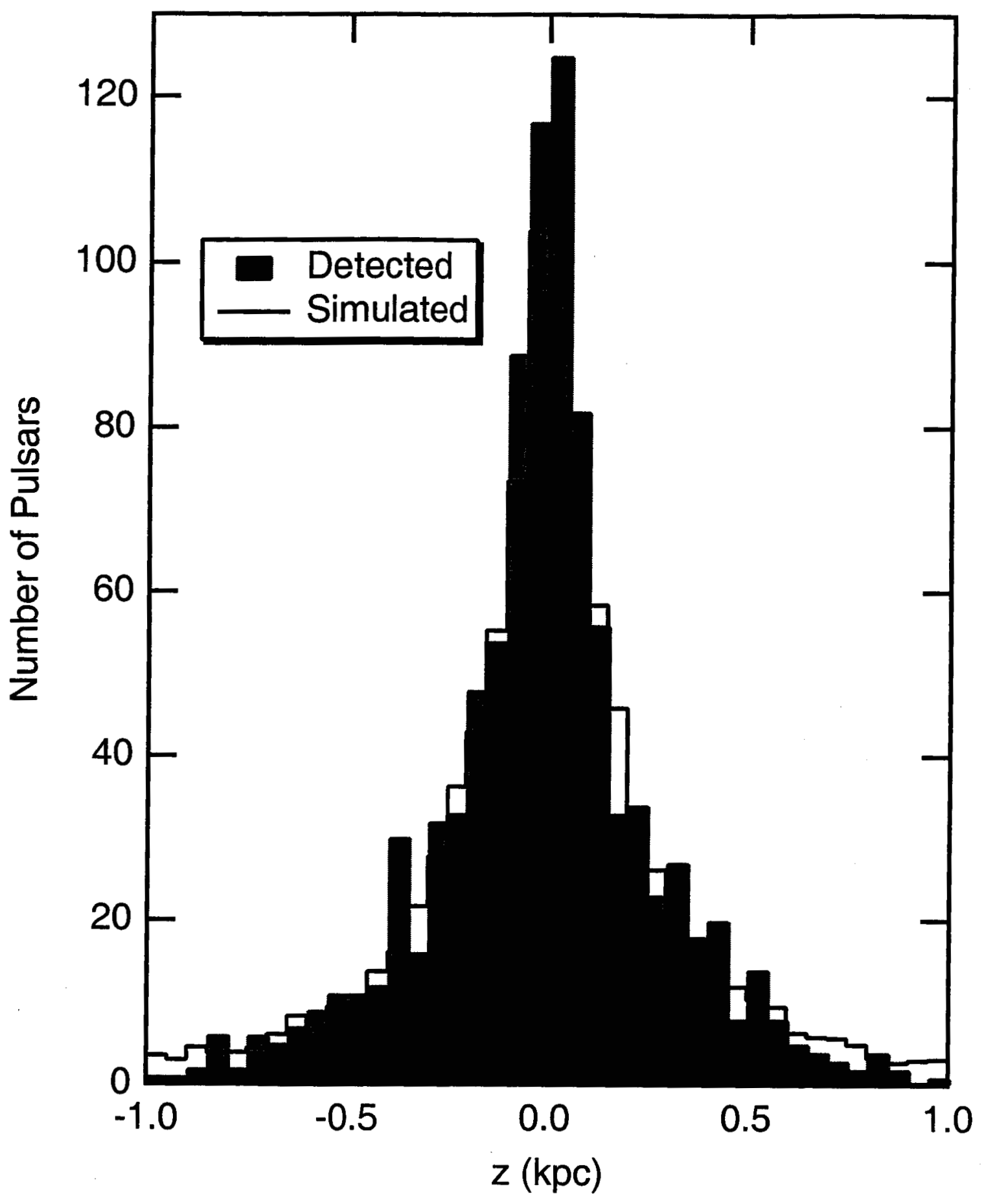

Fig. 6.- Distribution of the distance $\mathrm{z}$ from the Galactic disk for detected pulsars (shaded histogram) and simulated pulsars (regular histogram). 


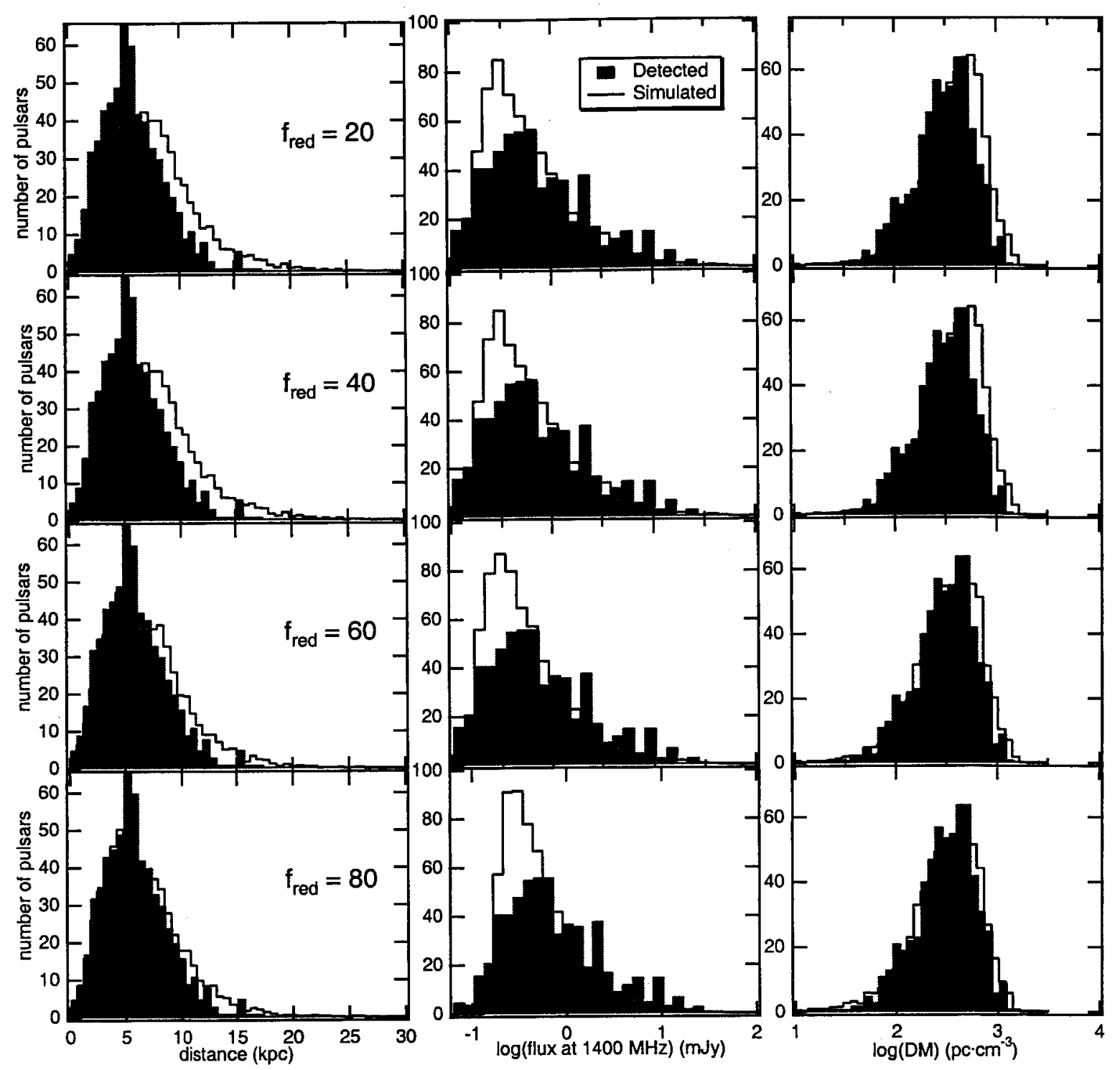

Fig. 7.- Distributions of the distance, flux at $1400 \mathrm{MHz}$ and dispersion measure for detected pulsars in the PMBPS (solid histograms) and simulated pulsars (regular histograms) for the indicated factor, $f_{\text {red }}$, reducing the radio luminosity of the ACC model. 


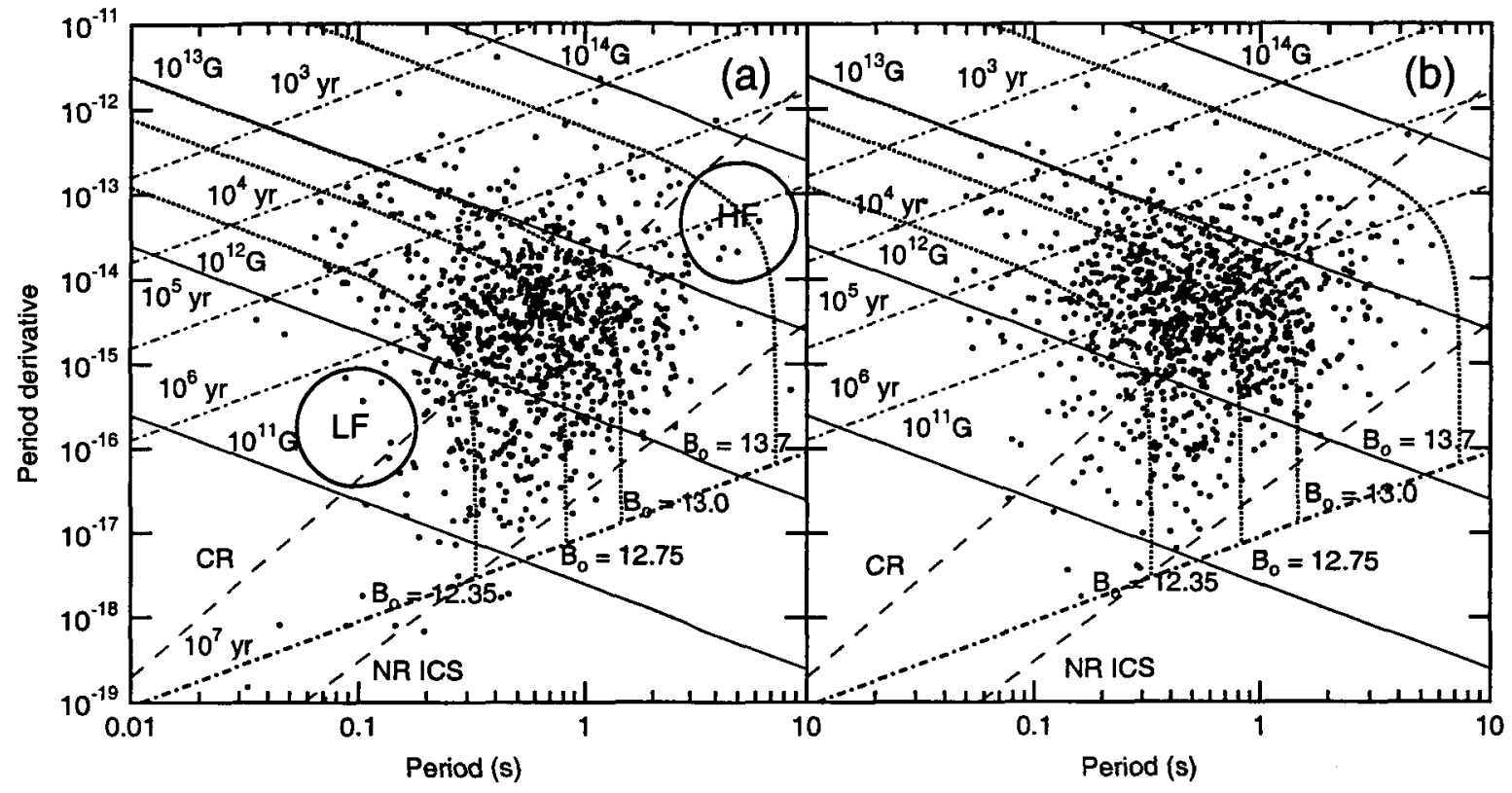

Fig. 8.- Distributions of (a) detected and (b) simulated pulsars with a field-decay constant of 2.8 Myr. Dashed lines represent the death lines for curvature radiation (upper) and for nonresonant inverse Compton scattering (lower). Dot-dashed lines represent the indicated pulsar age assuming a field decay of $2.8 \mathrm{Myr}$ and solid lines represent the indicated magnetic surface field strength assuming a constant dipole spin-down field. Dotted curves for the indicated field strengths assume a decay constant of $2.8 \mathrm{Myr}$ of the magnetic field. Two regions of pulsar paucity are indicated by circles - one at low field (LF) and another at high field (HF). 

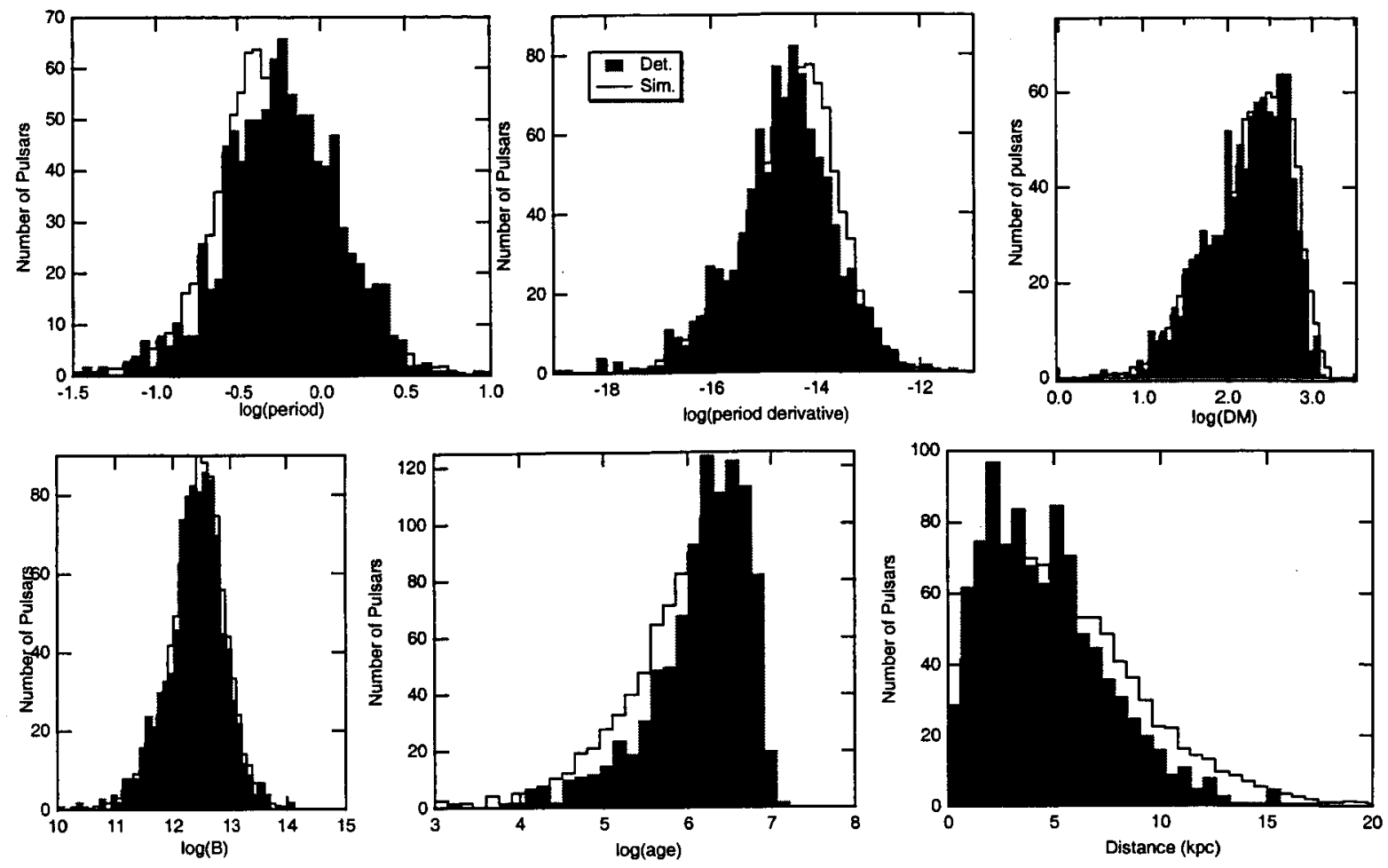

Fig. 9.- Distributions of various pulsar characteristics indicated as shaded histograms (detected pulsars) and plain histograms (simulated pulsars).
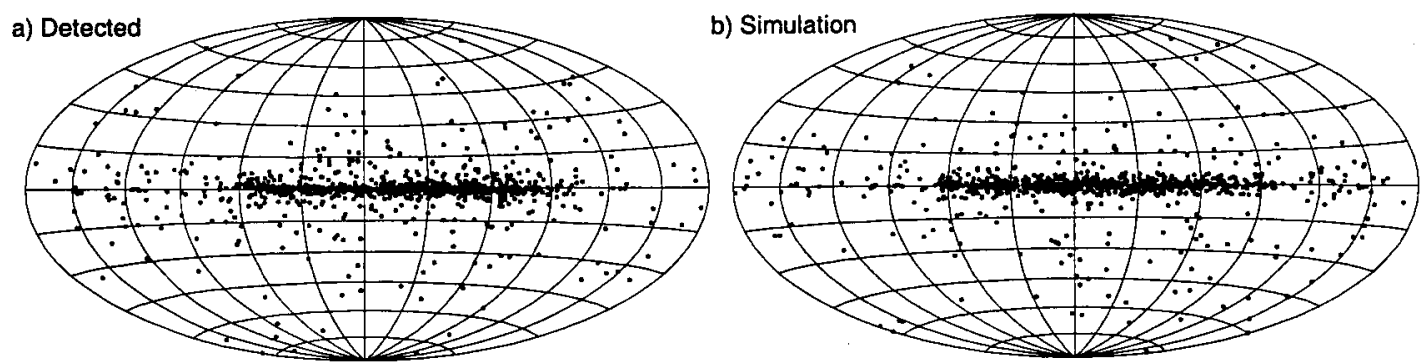

Fig. 10.- Aitoff plots of the (a) detected and (b) simulated radio pulsars. 


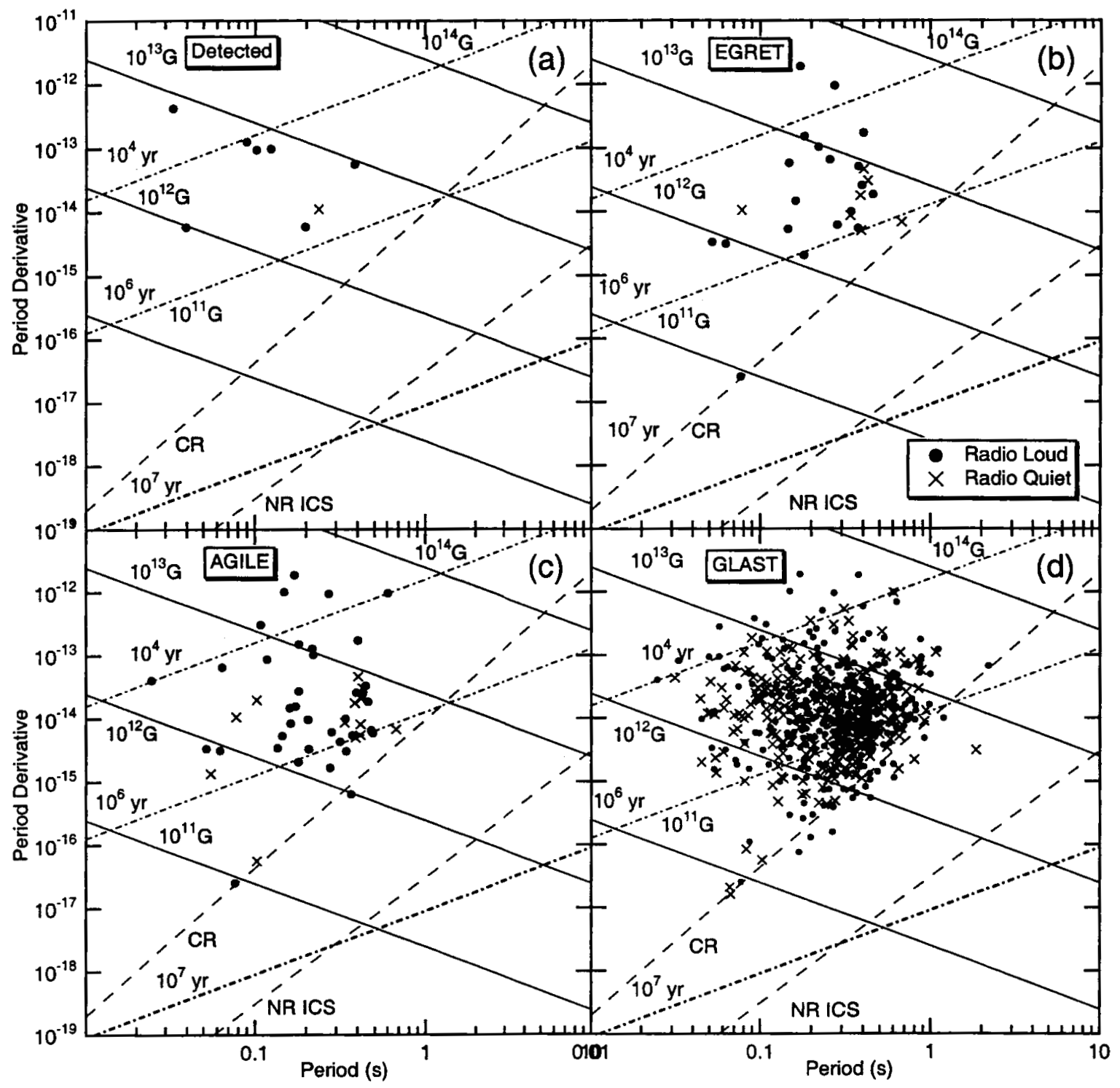

Fig. 11. - Distributions of radio-quiet (crosses) and radio-loud (solid dots) $\gamma$-ray pulsars (a) detected by EGRET and simulated for (b) EGRET (c) AGILE and (d) GLAST, assuming a field-decay constant of $2.8 \mathrm{Myr}$. Dashed lines represent the death lines for curvature radiation $(\mathrm{CR})$ and for nonresonant inverse Compton scattering (NRICS). Dot-dashed lines represent the indicated pulsar age assuming a field decay of $2.8 \mathrm{Myr}$ and solid lines represent the indicated magnetic surface field strength assuming a constant dipole spin-down field. 


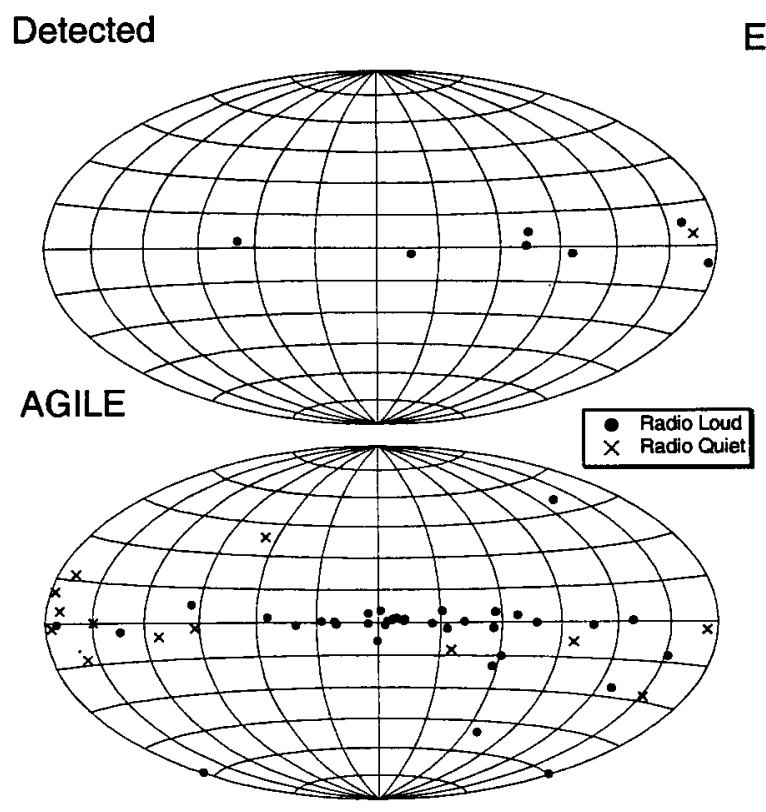

\section{EGRET}
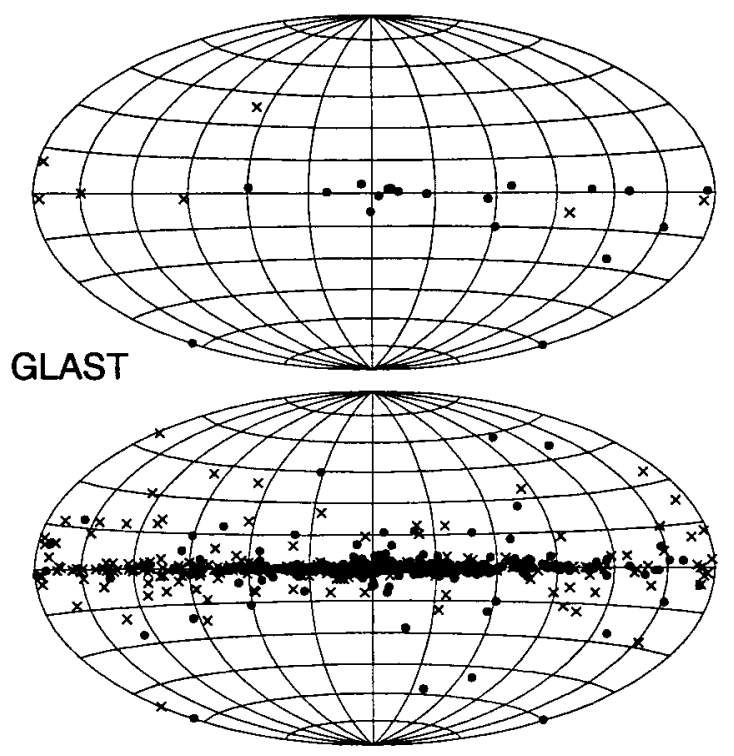

Fig. 12.- Aitoff plots of radio-quiet (crosses) and radio-loud (solid dots) $\gamma$-ray pulsars (a) detected by EGRET and simulated for (b) EGRET (c) AGILE and (d) GLAST, assuming a field-decay constant of $2.8 \mathrm{Myr}$. 


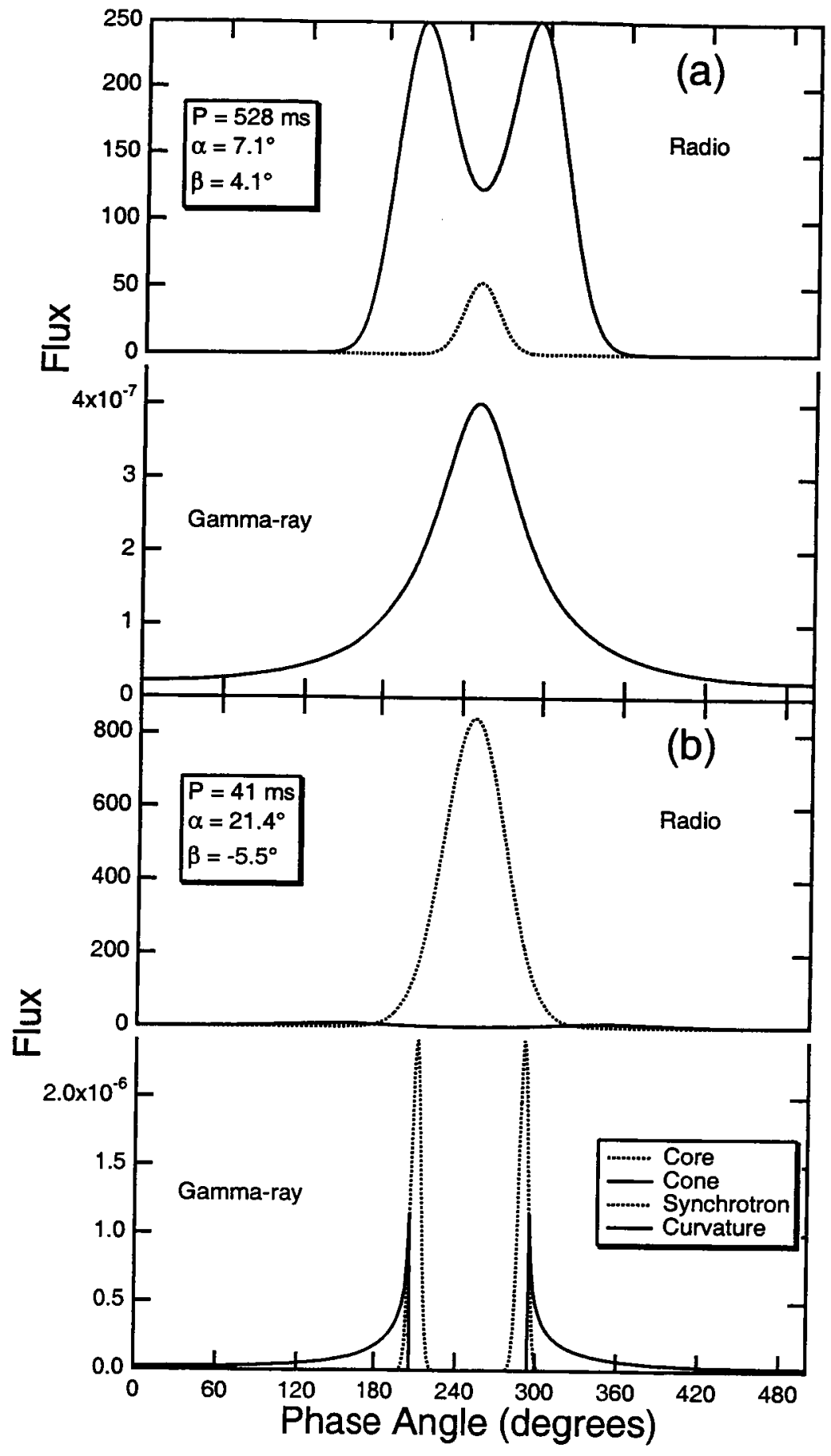

Fig. 13. - Examples of radio and $\gamma$-ray pulse profiles for two radio-loud $\gamma$-ray pulsars simulated for EGRET having periods of $528 \mathrm{~ms}$ (a) and $41 \mathrm{~ms}$ (b) 